\title{
A SPECTRAL DECOMPOSITION FOR SELF-ADJOINT ELEMENTS IN THE MAXIMUM GCR IDEAL OF A VON NEUMANN ALGEBRA WITH APPLICATIONS TO NONCOMMUTATIVE INTEGRATION THEORY $\left(^{1}\right)$
}

\author{
BY \\ HERBERT HALPERN
}

1. Introduction. Let $\mathscr{A}$ be a von Neumann algebra of Type I with center $\mathscr{Z}$ and let $I_{a}$ be the ideal in $\mathscr{A}$ generated by the abelian projections of $\mathscr{A}$. The ideal $I_{a}$ is the natural analogue in a von Neumann algebra of the ideal of completely continuous operators on a Hilbert space. Here, however, the center $\mathscr{Z}$ functions in the same way that the complex numbers function in the case of completely continuous operators. In fact, we prove that every positive operator $A$ in $I_{a}$ may be written in the form $A=\sum\left\{A_{j} E_{j} \mid j=1,2, \ldots\right\}$ where $\left\{E_{j} \mid j=1,2, \ldots\right\}$ is a sequence of mutually orthogonal abelian projections such that $E_{1}>E_{2} \succ \cdots$ and $\left\{A_{j} \mid j=1,2, \ldots\right\}$ is a sequence of positive central elements such that $A_{1} \geqq A_{2} \geqq \cdots$ and $\lim _{j} A_{j}=0$. This decomposition for $A$ will be unique in the same sense that the spectral decomposition for completely continuous operators is unique.

The ideal $I_{a}$ is the maximum $G C R$ ideal in the von Neumann algebra $A$. In fact, $I_{a}$ is a $C C R$ ideal and it has a Hausdorff structure space. In $\$ 3$ we show that the structure space of $I_{a}$ can be identified with the spectrum of the center $\mathscr{Z}$. Then we describe the positive elements $A$ in $I_{a}$ and in $\mathscr{A}$ with continuous trace i.e. those positive elements $A$ such that $\phi \rightarrow$ Trace $(\phi(A))$ is a continuous function from the space of equivalence classes of irreducible representations of $I_{a}$ and $\mathscr{A}$ respectively to the complex field.

In $\S 4$ we define in $\mathscr{A}$ analogues $\mathscr{S}$ and $\mathscr{T}$ to the Schmidt class and trace class in the algebra of all bounded linear operators on a Hilbert space. The sets $\mathscr{S}$ and $\mathscr{T}$ are ideals of $\mathscr{A}$ contained in $I_{a}$ and $\mathscr{S}^{2}=\mathscr{T}$. Furthermore, a trace $\operatorname{Tr}$ (with values in $\mathscr{Z})$ is defined on $\mathscr{T}$. This trace can be extended to a normal trace on $\mathscr{A}^{+}$. The ideal $\mathscr{S}$ under an inner product defined by $\operatorname{Tr}$ becomes a $\mathscr{Z}$-module [9] and the set of all uniformly continuous functions on $I_{a}$ into $\mathscr{Z}$ that are linear over $\mathscr{Z}$ is identified with $\mathscr{T}$. Also, a norm equal to the operator bound on $\mathscr{T}$ gives a Banach *-algebra structure on $\mathscr{T}$. With this norm the set of all continuous functions of $\mathscr{T}$ into $\mathscr{Z}$ that are linear over $\mathscr{Z}$ is identified with $\mathscr{A}$ and the operator bound is the norm defined on $\mathscr{A}$. The positive elements in $\mathscr{T}$ are identified with the normal function of $\mathscr{A}$ into $\mathscr{Z}$ which are linear over $\mathscr{Z}$. These are analogues of results

Received by the editors May 4, 1967.

(1) This research was partially supported by an Illinois Institute of Technology faculty research grant. 
concerning scalar valued traces on von Neumann algebras and results on completely continuous operators on Hilbert space.

Finally, we justify the use of the name Schmidt class and trace class by showing that these ideals contain all other ideals which produce $\mathscr{Z}$-modules. We also show the way in which this theory differs from the usual noncommutative integration theory.

2. Diagonalization of self-adjoint operators in $I_{a}$. Let $\mathscr{A}$ be a Type I von Neumann algebra with center $\mathscr{Z}$ and let $Z$ be the spectrum of $\mathscr{Z}$. For each $\zeta \in Z$ define $[\zeta]$ to be the closed two-sided ideal given by

$$
[\zeta]=\text { closure }\left\{\sum\left\{A_{j} B_{j} \mid 1 \leqq j \leqq n\right\} \mid A_{j} \in A, B_{j} \in \zeta, n \text { a positive integer }\right\} \text {. }
$$

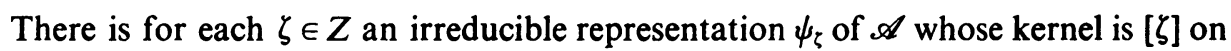
the Hilbert space $H(\zeta)$. We denote the image of $A$ in $\mathscr{A}$ under $\psi_{\zeta}$ by $A(\zeta)$. Then the function $\zeta \rightarrow\|A(\zeta)\|$ of $Z$ into the positive real numbers is a continuous function [4, $\$ 4$ ]. The image of $I_{a}$ under $\psi_{\zeta}$ is the ideal of all completely continuous operators of $H(\zeta)$. If $\mathscr{A}$ is homogeneous of finite degree $n$, the Hilbert space $H(\zeta)$ is $n$-dimensional.

We shall use the following terminology. Let $\mathscr{Z}$ be a commutative von Neumann algebra with spectrum $Z$. The projection in $\mathscr{Z}$ whose image under the Gelfand isomorphism $A \rightarrow A^{\wedge}$ of $\mathscr{Z}$ onto the set of continuous complex-valued functions on $Z$ is the characteristic function of an open and closed set in $W$ in $Z$ will be called the projection corresponding to $W$.

In order to obtain the decomposition for self-adjoint elements in $I_{a}$ we need the following lemma.

Lemma. Let $\mathscr{A}$ be a finite Type I von Neumann algebra which has only finitely many homogeneous components of distinct degrees. Let $\mathscr{Z}$ be the center of $\mathscr{A}$ and let $Z$ be the spectrum of $\mathscr{Z}$. Let $A$ be a self-adjoint element of $\mathscr{A}$ and let $A_{1}$ be an element in $\mathscr{Z}$ such that for each $\zeta$ in $Z$ the number $A_{1}(\zeta)$ is a proper value of the matrix $A(\zeta)$ on the finite-dimensional Hilbert space $H(\zeta)$. Then there is an abelian projection $E$ in $\mathscr{A}$ of central support 1 such that $A E=A_{1} E[1]$.

The following theorem allows us to apply the results of this lemma.

TheOREM 2.1. Let $\mathscr{A}$ be a Type I von Neumann algebra on the Hilbert space $H$; a projection $E$ in the ideal $I_{a}$ generated by the abelian projections of $\mathscr{A}$ is the sum of a finite number of mutually orthogonal abelian projections.

Proof. The algebra $E \mathscr{A} E$ is a Type I von Neumann algebra on $E(H)$. If $\phi$ is an irreducible representation of $E \mathscr{A} E$ on a Hilbert space $K$, then $K$ is finite dimensional. Indeed, there is an irreducible representation $\phi^{\prime}$ of $\mathscr{A}$ on a Hilbert space $K^{\prime}$ which contains $K$ as a subspace such that $\phi^{\prime}(E)$ is the projection corresponding to $K$ and $\phi^{\prime}(A) x=\phi(A) x$ for every $x \in K$ and $A \in E \mathscr{A} E$. We have that $\phi^{\prime}\left(I_{a}\right)$ is the set of 
completely continuous operators on $K^{\prime}$. This means that $\phi^{\prime}(E)$ is a finite-dimensional projection and, therefore, that $K$ is finite dimensional.

Let $\left\{P_{n}\right\}$ be a net of mutually orthogonal projections in the center of $E \mathscr{A} E$ such that the least upper bound of the $P_{n}$ is $E$ and $E \mathscr{A} E P_{n}$ is homogeneous. Each $E \mathscr{A} E P_{n}$ must have finite degree and so we may assume $\left\{P_{n}\right\}$ is either a finite set or an infinite sequence such that if $f(n)$ is the degree of $E \mathscr{A} E P_{n}$ then $f(n)<f(n+1)$ $(n=1,2, \ldots)$. It is sufficient to prove that the set $\left\{P_{n}\right\}$ is a finite set. We argue by contradiction. If there are an infinite number of $\left\{P_{n}\right\}$ there is a point $\zeta$ in the spectrum of the center of $E \mathscr{A} E$ such that $P_{n}^{\wedge}(\zeta)=0$ for all $n$. Let $\left\{E_{n j} \mid 1 \leqq j \leqq f(n)\right\}$ be mutually orthogonal equivalent abelian projections of sum $P_{n}$. Let $F_{j}=$ $\sum\left\{E_{n j} \mid k+1 \leqq n\right\}$ whenever $f(k)+1 \leqq j \leqq f(k+1)$ for $k=0,1,2, \ldots$ where $f(0)=0$. Each $F_{j}$ is an abelian projection; if $f(k)+1 \leqq j \leqq f(k+1)$ the central support of $F_{j}$ is $\sum\left\{P_{n} \mid k+1 \leqq n\right\}$. We have that $\left(\sum\left\{P_{n} \mid n \leqq k\right\}\right)^{\wedge}(\zeta)=0$ and so $\left(\sum\left\{P_{n} \mid k+1 \leqq n\right\}\right)^{\wedge}(\zeta)$

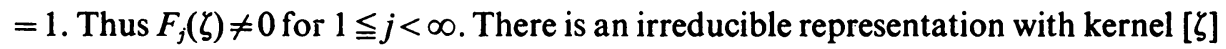
of $E \mathscr{A} E$ on a Hilbert space $H(\zeta)$. This means that $\sum\left\{F_{j}(\zeta) \mid 1 \leqq j \leqq f(k)\right\} \leqq E(\zeta)$ for all $k$. Because $\left\{F_{j}(\zeta)\right\}$ is a sequence of nonzero mutually orthogonal projections, $H(\zeta)$ must be infinite dimensional. This is impossible. So there are only finitely many $P_{n}$.

We now apply Lemma and Theorem 2.1 to the spectral decomposition of a selfadjoint operator in $I_{a}$ to obtain a diagonalization. By a rearrangement of this diagonalization we prove a spectral theorem analogous to the spectral theorem for self-adjoint completely continuous operators.

THEOREM 2.2. Let $\mathscr{A}$ be a Type I von Neumann algebra with center $\mathscr{Z}$ and let $I_{a}$ be the ideal generated by the abelian projections of $\mathscr{A}$. Let $A$ be a positive element in $I_{a}$. There is an at most denumerable set $\left\{E_{n}\right\}$ of mutually orthogonal abelian projections such that $E_{1} \succ E_{2} \succ \cdots$ and at most denumerable set $\left\{A_{n}\right\}$ of positive elements in $\mathscr{Z}$ such that $A_{1} \geqq A_{2} \geqq \cdots$ and such that $\lim _{n} A_{n}=0$ if there are infinitely many $A_{n}$ with the property $A=\sum_{n} A_{n} E_{n}\left({ }^{2}\right)$.

Proof. Let $Z$ be the spectrum of the center $\mathscr{Z}$. Because the function $\zeta \rightarrow\|A(\zeta)\|$ of $Z$ into the real numbers is continuous, there is an element $A_{1}$ in $\mathscr{Z}^{+}$such that $A_{1}^{\hat{i}}(\zeta)=\|A(\zeta)\|$ for every $\zeta \in Z$. There is no loss of generality in the assumption that $\|A\|=1$. Let $X_{n}^{\prime}=$ closure $\left\{\zeta \in Z \mid A_{1}^{\wedge}(\zeta)>(n+1)^{-1}\right\}$ for $n=1,2, \ldots$ The sets $X_{n}^{\prime}$ are open and closed subsets of $Z$. By induction we may define the sets $X_{1}=X_{1}^{\prime}$ and $X_{j}=X_{j}^{\prime}-\bigcup\left\{X_{k} \mid 1 \leqq k<j\right\}$ for $j=2,3, \ldots$ The sets $X_{n}$ are mutually disjoint and are open and closed. Let $P_{n}$ be the central projection which corresponds to $X_{n}$ for each $n=1,2, \ldots$.

Let $n$ be a fixed natural number and let $B=A P_{n}$. Let $\mathscr{B}$ be a maximal commutative *-subalgebra of $\mathscr{A} P_{n}$ that contains $B$ and let $Z(\mathscr{B})$ denote the spectrum of $\mathscr{B}$. Let $E$ be the projection in $\mathscr{B}$ which corresponds to the open and closed set closure $\left\{\zeta^{\prime} \in Z(\mathscr{B}) \mid B^{\wedge}\left(\zeta^{\prime}\right)>(2(n+1))^{-1}\right\}$. The spectral projection $E$ of $B$ has the property

$\left.{ }^{2}\right)$ I wish to thank the referee for suggesting that a proof along the following lines might be possible. I had originally deduced the theorem from the lemma in a more computative manner. 
$B \geqq(2(n+1))^{-1} E$ and $B(1-E) \leqq(2(n+1))^{-1}(1-E)$. The central support of $E$ in $\mathscr{A}$ is $P_{n}$. Indeed, if $E(\zeta)=0$ for some $\zeta \in X_{n}$, then $\|B(\zeta)\|=\|B(1-E)(\zeta)\| \leqq(2(n+1))^{-1}$. This is impossible. Thus $E(\zeta) \neq 0$ for every $\zeta \in X_{n}$. Now $B \geqq(2(n+1))^{-1} E$ implies that there is an element $C$ in $\mathscr{B}$ such that $C B=E$. Therefore $E$ is a projection in $I_{a}$. We have that $\|B(\zeta)\|=\max \{\|B E(\zeta)\|,\|B(1-E)(\zeta)\|\}$ for every $\zeta \in X_{n}$. Since

$$
\|B(1-E)(\zeta)\| \leqq(2(n+1))^{-1}
$$

we find that $\|B(\zeta)\|=\|B E(\zeta)\|$ for every $\zeta \in X_{n}$. Let $B^{\prime}=B E$. By Theorem 2.1 the Type I algebra $\mathscr{A}_{E}$ is finite with finitely many homogeneous components of distinct degrees. Let $Z\left(\mathscr{A}_{E}\right)$ be the spectrum of the center of $\mathscr{A}_{E}$. The function $\zeta^{\prime \prime} \rightarrow\left\|B^{\prime}\left(\zeta^{\prime \prime}\right)\right\|$ is continuous on $Z\left(\mathscr{A}_{E}\right)$ and there is an element $B_{1}$ in the center of $\mathscr{A}_{E}$ such that $B_{1}\left(\zeta^{\prime \prime}\right)=\left\|B^{\prime}\left(\zeta^{\prime \prime}\right)\right\|$ for every $\zeta^{\prime \prime} \in Z\left(\mathscr{A}_{E}\right)$.

There is an abelian projection $F=F_{n}$ in $\mathscr{A}_{E}$ of central support $E$ such that $B F=B_{1} F$. Considering $F$ as a projection of $\mathscr{A}$, we see that $F$ is an abelian projection of $\mathscr{A}$ of central support $P_{n}$. The center of $\mathscr{A}_{E}$ is $\mathscr{Z} E$ and the function $\rho(C)=C E$ of $\mathscr{Z} P_{n}$ onto $\mathscr{Z} E$ is an isomorphism. Let $A_{1}^{\prime}$ be the unique element in $\mathscr{Z} P_{n}$ such that $A_{1}^{\prime} E=B_{1}$; we show that $A_{1}^{\prime}=A_{1} P_{n}$. Let $\zeta \in X_{n}$ and let $\zeta^{\prime}$ be the maximal ideal in $\mathscr{Z} P_{n}$ given by $\zeta^{\prime}=\left\{C P_{n} \mid C \in \zeta\right\}$; then $A_{1}^{\prime}(\zeta)=B_{1}^{\wedge}\left(\rho\left(\zeta^{\prime}\right)\right)=\left\|B^{\prime}\left(\rho\left(\zeta^{\prime}\right)\right)\right\|=$ $\inf \left\{\left\|B^{\prime}+D\right\| \mid D \in\left[\rho\left(\zeta^{\prime}\right)\right]\right\} \geqq \inf \left\{\left\|B^{\prime}+D\right\| \mid D \in[\zeta]\right\}=\|B(\zeta)\|=A_{1}(\zeta)$ because the ideal $\left[\rho\left(\zeta^{\prime}\right)\right]$ in $\mathscr{A}_{E}$ is contained in the ideal $[\zeta]$ in $\mathscr{A}$. So $A_{1}^{\prime} \geqq A_{1} P_{n}$. However, if $\zeta \in X_{n}$ and if $D \in[\zeta]$ it is easy to see that $E D E \in\left[\rho\left(\zeta^{\prime}\right)\right]$. This means that $A_{1}^{\prime \wedge}(\zeta)=\left\|B^{\prime}\left(\rho\left(\zeta^{\prime}\right)\right)\right\|$ $\leqq\|E B E-E D E\| \leqq\|B-D\|$, for every $D \in[\zeta]$. Thus, $A_{1}^{\prime}(\zeta) \leqq\|B(\zeta)\|=A_{1}(\zeta)$ for every $\zeta \in X_{n}$. So $A_{1}^{\prime} \leqq A_{1} P_{n}$. Consequently, we have found an abelian projection $F_{n}$ of central support $P_{n}$ such that $A F_{n}=A_{1} F_{n}$.

Let $P=\sum\left\{P_{n} \mid n=1,2, \ldots\right\}$ and let $E_{1}=\sum\left\{F_{n} \mid n=1,2, \ldots\right\}$; the projection $E_{1}$ is abelian and has central support $P$ which corresponds to the closure of the set $\{\zeta \in Z \mid\|A(\zeta)\| \neq 0\}$. Furthermore, $A E_{1}=A_{1} E_{1}$. Then the operator $A\left(1-E_{1}\right)$ is a positive operator in $I_{a}$ and for every $\zeta$ in $Z$ the relation $\left\|A\left(1-E_{1}\right)(\zeta)\right\| \leqq\|A(\zeta)\|$ - $\left\|\left(1-E_{1}\right)(\zeta)\right\| \leqq A_{1}(\zeta)$ is true. By induction we can construct a sequence $\left\{A_{n}\right\}$ of positive central elements such that $A_{1} \geqq A_{2} \geqq \cdots$ and a sequence of mutually orthogonal abelian projections $\left\{E_{n} \mid n=0,1,2, \ldots\right\}$ such that

(i) $E_{0}=0$;

(ii) $A E_{n}=A_{n} E_{n}(n=1,2, \ldots)$;

(iii) The function $\zeta \rightarrow\left\|A\left(1-\sum\left\{E_{j} \mid 0 \leqq j \leqq n-1\right\}\right)(\zeta)\right\|$ on $Z$ is equal to $A_{n}$ $(n=1,2, \ldots)$; and

(iv) The central support $Q_{n}$ of $E_{n}$ corresponds to the closure of the set

$$
\left\{\zeta \in Z \mid A_{n}^{\wedge}(\zeta) \neq 0\right\} .
$$

In view of (iii) in order to show $A=\sum_{n} A_{n} E_{n}$ (uniformly), it is sufficient to show that $\lim _{n} A_{n}=0$ (uniformly). Indeed,

$$
\left\|A-\sum\left\{A_{j} E_{j} \mid 1 \leqq j \leqq n\right\}\right\|=\left\|A\left(1-\sum\left\{E_{j} \mid 1 \leqq j \leqq n\right\}\right)\right\|=\left\|A_{n+1}\right\| .
$$


We prove $\lim _{n} A_{n}=0$ by arguing by contradiction. Suppose $\lim _{n} A_{n} \neq 0$. Taking into consideration the ordering of the sequence $\left\{A_{n}\right\}$, there is a number $\delta>0$ such that $\left\|A_{n}\right\|>\delta$ for every $n$. So for each $n$ there is a maximal ideal $\zeta_{n}$ in $Z$ such

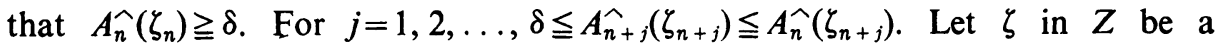
limit point of the set $\left\{\zeta_{n} \mid n=1,2, \ldots\right\}$. For every $j=1,2, \ldots$ we have that $A_{j}(\zeta) \geqq \lim \inf _{n} A_{j}\left(\zeta_{n}\right) \geqq \delta$. Because each $A_{j}(\zeta)$ is not zero, each $E_{j}(\zeta)$ is a onedimensional projection on the Hilbert space $H(\zeta)$. The sequence $\left\{E_{n}(\zeta)\right\}$ is a sequence of orthogonal one-dimensional projections on $H(\zeta)$ such that $A(\zeta) E_{j}(\zeta)=A_{j}(\zeta) E_{j}(\zeta)$ for each $j=1,2, \ldots$. This shows that the compact operator $A(\zeta)$ has infinitely many proper values which are greater than or equal to the strictly positive number $\delta$. This is impossible. Hence, we conclude $\lim _{n} A_{n}=0$.

Finally, if $Q_{n}$ is the central support of $E_{n}(n=1,2, \ldots)$, then $Q_{n}$ corresponds to the set closure $\left\{\zeta \in Z \mid A_{n}(\zeta) \neq 0\right\}$. So $Q_{1} \geqq Q_{2} \geqq \cdots$. Thus $E_{1} \succ E_{2} \succ \cdots$.

Using the spectral theorem for completely continuous self-adjoint operators on a Hilbert space, we prove that the representation obtained in Theorem 2.2 is unique.

THEOREM 2.3. Let $A$ be a positive element in $I_{a}$ and let $\left\{A_{j} \mid 1 \leqq j<m\right\}$ (respectively, $\left\{B_{j} \mid 1 \leqq j<n\right\}$ ) be a set of positive central elements and $\left\{E_{j} \mid 1 \leqq j<m\right\}$ (respectively, $\left\{F_{j} \mid 1 \leqq j<n\right\}$ ) be a set of orthogonal abelian projections with the following properties: (1) $A_{j} \neq 0$ (respectively, $B_{j} \neq 0$ ) for all $j$; (2) $A_{1} \geqq A_{2} \geqq \cdots$ (respectively, $\left.B_{1} \geqq B_{2} \geqq \cdots\right)$; (3) if $Z$ is the spectrum of the center, then

$$
\left\{\zeta \in Z \mid E_{j}(\zeta) \neq 0\right\}=\text { closure }\left\{\zeta \in Z \mid \hat{A_{j}}(\zeta) \neq 0\right\}
$$

(respectively, $\left\{\zeta \in Z \mid F_{j}(\zeta) \neq 0\right\}=$ closure $\left\{\zeta \in Z \mid B_{j}(\zeta) \neq 0\right\}$ ) for every $j ;(4)$ if $m=+\infty$ (respectively, $n=+\infty$ ), then $\lim _{j} A_{j}=0$ (respectively, $\left.\lim _{j} B_{j}=0\right) ;(5) \sum A_{j} E_{j}=A$ (respectively, $\sum B_{j} F_{j}=A$ ). Then $m=n$ and $A_{j}=B_{j}$ for every $j$.

Proof. For every $\zeta$ in $Z$ the element $A(\zeta)$ is a completely continuous operator on $H(\zeta)$. We have that $\sum \widehat{A_{j}}(\zeta) E_{j}(\zeta)=A(\zeta)=\sum B_{j}(\zeta) F_{j}(\zeta)$ on $H(\zeta)$ since for both sums $A$ is the uniform limit of the partial sums $\sum\left\{A_{j} E_{j} \mid 1 \leqq j \leqq n\right\}$ and $\sum\left\{B_{j} F_{j} \mid 1 \leqq j \leqq n\right\}$. However, $\left\{\hat{A_{j}}(\zeta)\right\}$ and $\left\{B_{j}(\zeta)\right\}$ are decreasing sets of positive numbers and the nonzero $E_{j}(\zeta)$ and $F_{j}(\zeta)$ are one-dimensional projections on $H(\zeta)$. Because $E_{j}(\zeta)=0$ (respectively, $F_{j}(\zeta)=0$ ) implies $E_{k}(\zeta)=0$ (respectively, $\left.F_{k}(\zeta)=0\right)$ whenever $j \leqq k$, we have by the spectral theorem on $H(\zeta)$ that $A_{j}(\zeta) \neq 0$ if and only if $B_{j}(\zeta) \neq 0$ and that $A_{j}(\zeta)=B_{j}(\zeta)$ for all $j$ such that $A_{j}(\zeta) \neq 0$. Thus, we have the result that $m=n$ and $A_{j}(\zeta)=B_{j}(\zeta)$ for all $\zeta \in Z$. Thus, $A_{j}=B_{j}$ for all $j$. Q.E.D.

We call the representation for a self-adjoint operator $A$ in Theorem 2.2 a spectral representation for $A$.

3. Elements with continuous trace. Let $\mathscr{A}$ be a $C^{*}$-algebra and let $P(\mathscr{A})$ be the set of all primitive ideals of $\mathscr{A}$ with the hull-kernel topology. The space $P(\mathscr{A})$ is called the structure space of $\mathscr{A}$. Let $\mathscr{A}^{\wedge}$ be the set of all equivalence classes of irreducible representations of $\mathscr{A}$ with the reciprocal topology induced by the function $\phi \rightarrow$ kernel $\phi$ of $\mathscr{A}^{\wedge}$ into $P(\mathscr{A})$. For each $A$ in $\mathscr{A}^{+}$the trace of the image of $A$ under any 
of the representations in a single equivalence class $\phi$ in $\mathscr{A}^{\wedge}$ is invariant; the trace is denoted by $\operatorname{Tr}(\phi(A))$. If $A$ is an element in $\mathscr{A}^{+}$such that $\operatorname{Tr}(\phi(A))$ is finite for all $\phi \in \mathscr{A}^{\wedge}$ and such that $\phi \rightarrow \operatorname{Tr}(\phi(A))$ is continuous on $\mathscr{A}^{\wedge}$, then $A$ is said to have a continuous trace. The set of all positive elements with continuous trace is the set of all positive elements of a two-sided ideal in $m(\mathscr{A})$ of $\mathscr{A}[3, \S 4]$. In this section we study the elements with continuous trace in a von Neumann algebra.

We first prove a theorem concerning the structure space of $I_{a}$.

THEOREM 3.1. Let $\mathscr{A}$ be a Type I von Neumann algebra and let $\mathscr{Z}$ be the center of $\mathscr{A}$; let $Z$ be the spectrum of $\mathscr{Z}$. The structure space $P_{a}=P\left(I_{a}\right)$ is homeomorphic to $Z$ under the map $f(\zeta)=[\zeta] \cap I_{a}$.

Proof. The set $P_{a}$ is equal to $\left\{[\zeta] \cap I_{a} \mid \zeta \in Z\right\}$. Therefore $f$ is a surjection. We prove that $f$ is an injection and that $f$ is bicontinuous.

Suppose $\zeta_{1}$ and $\zeta_{2}$ are distinct elements in $Z$. There is a projection $P$ in $\mathscr{Z}$ such that $P^{\wedge}\left(\zeta_{1}\right)=1$ and $P^{\wedge}\left(\zeta_{2}\right)=0$. Let $E$ be an abelian projection of central support $P$; then $E\left(\zeta_{1}\right) \neq 0$ and $E\left(\zeta_{2}\right)=0$. Now for any $A$ in $I_{a}$ and any $\zeta \in Z$ we have that $\|A(\zeta)\|=\left\|A\left([\zeta] \cap I_{a}\right)\right\|$; thus, $E \notin f\left(\zeta_{1}\right)$ and $E \in f\left(\zeta_{2}\right)$ so $f\left(\zeta_{1}\right) \neq f\left(\zeta_{2}\right)$. This shows that $f$ is an injection.

We now show that $f$ is continuous. Let $X$ be an open set in $P_{a}$. Then there is a closed two-sided ideal $I$ in $I_{a}$ such that $X=\left\{J \in P_{a} \mid J \downarrow I\right\}$. We show that the set $\{\zeta \in Z \mid f(\zeta) \in X\}$ is open in $Z$. Indeed, we have that $f(\zeta) \neq I$ if and only if $[\zeta] \ngtr I$. But if $\left[\zeta_{0}\right] \ngtr I$ there is a projection $F$ in $I$ such that $\left\|F\left(\zeta_{0}\right)\right\|=1$. The set $\{\zeta \in Z \mid\|F(\zeta)\|=1\}$ is open in $Z$ and contains $\left[\zeta_{0}\right]$ and so $\{\zeta \in Z \mid f(\zeta) \ngtr I\}$ is open in $P_{a}$. Thus $f$ is continuous.

Let $Y$ be a closed set in $Z$. The set $Y$ is compact and, therefore, $f(Y)$ is compact in the Hausdorff space $P_{a}$. So $f(Y)$ is closed in $P_{a}$. This proves $f$ is closed and that $f^{-1}$ is continuous. Q.E.D.

Because $I_{a}$ is a $C C R$ algebra, the space $I_{a}^{\hat{a}}$ is homeomorphic to $P_{a}$ under the mapping $\phi \rightarrow \operatorname{kernel} \phi$. If $\psi$ is an irreducible representation whose equivalence class is $(\psi)$ the function $\zeta \rightarrow\left(\psi_{\zeta}\right)$ is a homeomorphism of $Z$ onto $I_{a}^{\wedge}$.

We are now ready to characterize $m\left(I_{a}\right)^{+}$. Let $\mathscr{Q}$ be the set of all positive elements $A$ in $I_{a}$ such that if $\sum A_{j} E_{j}$ is a spectral resolution of $A$ then $\sum A_{j}$ converges uniformly in $\mathscr{Z}$. In particular $\mathscr{Q}$ contains every projection of $I_{a}$. By Theorem 2.3 the sum $\sum A_{j}$ depends only on $A$. Define the trace $\operatorname{Tr}(A)$ of $A$ to be $\sum A_{j}$.

THEOREM 3.2. Let $\mathscr{A}$ be a Type I von Neumann algebra and let $\mathscr{Z}$ be the center of $\mathscr{A}$. Let $I_{a}$ be the ideal in $\mathscr{A}$ generated by the abelian projections of $\mathscr{A}$. The set $m\left(I_{a}\right)^{+}$of positive elements of $I_{a}$ with continuous trace is equal to the set $\mathscr{Q}$. Furthermore, if $A \in \mathscr{Q}, \operatorname{Tr}(A)^{\wedge}(\zeta)=\operatorname{Tr}\left(\psi_{\zeta}(A)\right)$ for each $\zeta$ in the spectrum $Z$ of $\mathscr{Z}$.

Proof. Let $A$ be an element in $\mathscr{Q}$ and let $\sum A_{j} E_{j}$ be a spectral representation of $A$. For each $\phi=\left(\psi_{\zeta}\right)$ in $I_{a}^{\wedge}$ where $\zeta \in Z$ we see that $\operatorname{Tr}(\phi(A))=\sum A_{j}^{\wedge}(\zeta)=\operatorname{Tr}(A)^{\wedge}(\zeta)$. Since $Z$ is homeomorphic to $I_{a}^{\wedge}$ under the mapping $\zeta \rightarrow\left(\psi_{\xi}\right), \phi \rightarrow \operatorname{Tr}(\phi(A))$ is 
continuous on $I_{a}^{\wedge}$. Indeed, this function is the uniform limit of continuous functions. So $\mathscr{Q} \subset m\left(I_{a}\right)^{+}$.

Conversely, suppose that $A \in m\left(I_{a}\right)^{+}$and let $\sum A_{j} E_{j}$ be a spectral representation of $A$. Then the function $\zeta \rightarrow \sum A_{j}(\zeta)=g(\zeta)$ is continuous on $Z$ because $\operatorname{Tr}\left(\psi_{\zeta}(A)\right)$ $=\sum A_{j}(\zeta)$. For each $j$ let us assume $A_{j} \neq 0$. Then if $g_{k}=\sum\left\{A_{j} \mid 1 \leqq j \leqq k\right\}$, the sequence $\left\{g_{k}\right\}$ of monotonically increasing continuous functions converges pointwise to the continuous function $g$. By Dini's theorem $g$ is the uniform limit of the $g_{k}$ i.e. $\sum A_{j}$ converges uniformly. So $A \in \mathcal{Q}$. This proves $m\left(I_{a}\right)^{+} \subset \mathcal{Q}$.

Corollary. For each $A_{1}, A_{2} \in \mathscr{Q}$ and $C_{1}, C_{2} \in \mathscr{Z}^{+} C_{j} A_{j}(j=1,2)$ are elements of $\mathscr{Q}$ and $\operatorname{Tr}\left(C_{1} A_{1}+C_{2} A_{2}\right)=C_{1} \operatorname{Tr}\left(A_{1}\right)+C_{2} \operatorname{Tr}\left(A_{2}\right)$.

Proof. It is obvious that $C_{j} A_{j} \in \mathcal{Q}$. Also for each $\zeta \in Z$

$$
\begin{aligned}
\operatorname{Tr}\left(C_{1} A_{1}+C_{2} A_{2}\right)^{\wedge}(\zeta) & =\operatorname{Tr}\left(C_{1}^{\wedge}(\zeta) A_{1}(\zeta)+C_{2}^{\wedge}(\zeta) A_{2}(\zeta)\right) \\
& =C_{1}^{\wedge}(\zeta) \operatorname{Tr}\left(A_{1}(\zeta)\right)+C_{2}^{\wedge}(\zeta) \operatorname{Tr}\left(A_{2}(\zeta)\right) \\
& =\left(C_{1} \operatorname{Tr}\left(A_{1}\right)+C_{2} \operatorname{Tr}\left(A_{2}\right)\right)^{\wedge}(\zeta)
\end{aligned}
$$

Thus, $\operatorname{Tr}\left(\sum C_{j} A_{j}\right)=\sum C_{j} \operatorname{Tr}\left(A_{j}\right)$. We show that $m\left(I_{a}\right)$ is an ideal in $\mathscr{A}$.

THEOREM 3.3. The ideal $m\left(I_{a}\right)$ of elements in $I_{a}$ with continuous trace is an ideal in $\mathscr{A}$.

Proof. If $A \in \mathscr{A}$ and $A^{*} A \in I_{a}$ then $A \in I_{a}$. Thus, $J=\left\{A \in I_{a} \mid A^{*} A \in \mathscr{Q}\right\}=$ $\left\{A \in \mathscr{A} \mid A^{*} A \in \mathscr{Q}\right\}$. The set $\mathscr{Q}$ has the following properties: (1) $\mathscr{Q}+\mathscr{Q} \subset \mathscr{Q}$; (2) if $A \in \mathscr{A}$ and $A^{*} A \in \mathscr{Q}$ then $A A^{*} \in \mathscr{Q}$; and (3) if $B \in \mathscr{Q}, A \in \mathscr{A}$ and $0 \leqq A \leqq B$, then $A \in \mathscr{Q}$. So $J$ is a two-sided ideal in $\mathscr{A}$ and $m\left(I_{a}\right)=J^{2}$ is an ideal in $\mathscr{A}$. Q.E.D.

In the standard way $\operatorname{Tr}$ on $\mathscr{Q}$ may be extended to a function of $m\left(I_{a}\right)$ into $\mathscr{Z}$ such that $\operatorname{Tr}\left(C_{1} A_{1}+C_{2} A_{2}\right)=C_{1} \operatorname{Tr}\left(A_{1}\right)=C_{2} \operatorname{Tr}\left(A_{2}\right)$ for every $A_{1}, A_{2} \in m\left(I_{a}\right)$ and $C_{1}, C_{2} \in \mathscr{Z}$. For each $\zeta \in Z$ and $A \in m\left(I_{a}\right)$ it is true that $\operatorname{Tr}(A)^{\wedge}(\zeta)=\operatorname{Tr}(A(\zeta))$.

Lemma. Let $\mathscr{A}$ be a Type I von Neumann algebra with center $\mathscr{Z}$. Let $Z$ be the spectrum of $\mathscr{Z}$ and let $\zeta \in Z$. Let $\left\{x_{n}\right\}$ be a sequence of orthonormal vectors in $H(\zeta)$. There is a sequence of abelian projections $\left\{E_{n}\right\}$ in $\mathscr{A}$ such that $E_{n}>E_{n+1}$ and $E_{n}(\zeta) x_{n}$ $=x_{n}$ for all $n$.

Proof. This lemma is a simple extension of a result of Glimm [15, §5, Lemma 11]. Let $E_{1}$ be a projection in the ideal $I_{a}$ generated by the abelian projections such that $E_{1}(\zeta) x_{1}=x_{1}$. Indeed, $I_{a}(\zeta)$ is the ideal of completely continuous operators on $H(\zeta)$. Suppose $E_{1}, E_{2}, \ldots, E_{n}$ have been constructed. Let $F$ be a projection in $I_{a}$ such that $F(\zeta)$ is the projection of $H(\zeta)$ onto the subspace spanned by $x_{n+1}$ and such that $\left(E_{1}+E_{2}+\cdots+E_{n}\right)(\zeta) F(\zeta)=0$ in an open and closed neighborhood $W$ of $\zeta$. Let $F^{\prime}$ be an abelian projection whose central support $R$ is the same as the central support of $F$. Then $F^{\prime}(\zeta) \neq 0$ and so $F^{\prime}(\zeta)$ is the projection of $H(\zeta)$ on the subspace 
spanned by $x_{n+1}$. Let $P$ be the central support of $E_{n}$ and let $Q$ be the central projection corresponding to $W$. Then let $E_{n+1}=F^{\prime} P Q$. We have that $E_{n+1}$ is abelian and $E_{n+1}(\zeta)=F^{\prime}(\zeta)$. Also $E_{j} E_{n+1}=0$ for $j=1,2, \ldots, n$ and $E_{n}>E_{n+1}$ because the central support of $E_{n}$ majorizes that of $E_{n+1}$. Q.E.D.

THEOREM 3.4. Let $m\left(I_{a}\right)$ be the ideal of elements with continuous trace in the ideal generated by the abelian projections of a Type I von Neumann algebra $\mathscr{A}$. For every $\zeta$ in the spectrum $Z$ of the center of $\mathscr{A}$ the image $m\left(I_{a}\right)(\zeta)$ of $m\left(I_{a}\right)$ under the homeomorphism $\psi_{\zeta}$ of $\mathscr{A}$ with kernel $[\zeta]$ is the trace class of $H(\zeta)$.

Proof. Let $A \in \mathscr{Q}$. Then $\psi_{\zeta}(A)(\zeta \in Z)$ has a trace on $H(\zeta)$. So $A(\zeta)$ is of trace class on $H(\zeta)$. Thus $m\left(I_{a}\right)(\zeta)$ is contained in the trace class of $H(\zeta)$.

Now let $A$ be in the trace class of $H(\zeta)$. We may assume $A$ is positive. There is a sequence $\left\{x_{j}\right\}$ of orthonormal vectors in $H(\zeta)$ and a decreasing sequence of positive real numbers such that $A=\sum \alpha_{j} P_{j}$ where $P_{j}$ is the one-dimensional projection on the subspace spanned by $x_{j}$. There is a sequence of mutually orthogonal abelian projections $\left\{E_{j}\right\}$ in $\mathscr{A}$ such that $E_{j} \succ E_{j+1}($ all $j)$ and $E_{j}(\zeta)=P_{j}$. Then the operator $\sum \alpha_{j} E_{j}=B$ is in $I_{a}$ and if $R_{j}$ is the central support of $E_{j}, \sum\left(\alpha_{j} R_{j}\right) E_{j}$ is a spectral resolution for $B$. But $\sum\left\|\alpha_{j} R_{j}\right\|=\sum \alpha_{j}<+\infty$ and so $B \in \mathscr{Q}$. Furthermore, $B(\zeta)=A$. This proves $m\left(I_{a}\right)(\zeta)$ contains the trace class of $H(\zeta)$. Hence, $m\left(I_{a}\right)(\zeta)$ is the trace class of $H(\zeta)$. Q.E.D.

We now determine the set $m(\mathscr{A})^{+}$when $\mathscr{A}$ is a von Neumann algebra.

THeOREM 3.5. Let $\mathscr{A}$ be a von Neumann algebra with center $\mathscr{Z}$. For each $n$ $=1,2, \ldots$ let $R_{n}$ be the largest projection $P$ in $\mathscr{Z}$ such that $\mathscr{A} P$ is of Type $\mathrm{I}_{n}$. Let $R=\sum R_{n}$ and let $\mathscr{Q} R=\{A \in \mathscr{Q} \mid A(1-R)=0\}$. Then $m(\mathscr{A})^{+}$is equal to the set of all $A$ in $\mathscr{Q} R$ such that $\lim _{n} \operatorname{Tr}(A) R_{n}=0$.

Proof. The uniform closure $I$ of $m(\mathscr{A})$ is a uniformly closed two-sided ideal in $\mathscr{A}$; the space $I^{\wedge}$ is homeomorphic to the open subset $\left\{\phi \in \mathscr{A}^{\wedge} \mid\right.$ kernel $\left.\phi \nsupseteq I\right\}$ under the inverse of the map $(\psi) \rightarrow(\psi \mid I)$. Therefore $\phi \rightarrow \operatorname{Tr}(\phi(A))$ is continuous on $I^{\wedge}$ for all $A \in m(\mathscr{A})^{+}$. This shows that $I$ is a $C C R$ ideal in $\mathscr{A}[3, \S 4]$. Because $I_{a}$ is the maximal $G C R$ ideal in $\mathscr{A}$, we have that $I \subset I_{a}$. So if $P$ is the central projection of $\mathscr{A}$ such that $\mathscr{A} P$ is discrete and $\mathscr{A}(1-P)$ is continuous we have that $m(\mathscr{A}) \cdot(1-P)=0$.

Now we show that $m(\mathscr{A})(P-R)=(0)$. Let $A \in m(\mathscr{A})^{+}$. Assume $P-R \neq 0$. Let $Z$ be the spectrum of $\mathscr{Z}$ and let $X=\left\{\zeta \in Z \mid(P-R)^{\wedge}(\zeta)=1\right\}$. For each $\zeta \in X$ the ideal $I_{a}+[\zeta]$ is proper. Indeed, the representation $\psi_{\zeta}$ is an irreducible representation of $\mathscr{A}$ on $H(\zeta)$ such that $\psi_{\zeta}\left(I_{a}\right)$ is the ideal of completely continuous operators on $H(\zeta)$. But for any $n=1,2, \ldots, P-R$ may be written as the sum of $n$ equivalent projections; so $\psi_{\zeta}(1)$ does not have finite dimensions in $H(\zeta)$ and thus $\psi_{\zeta}(1) \notin \psi\left(I_{a}\right)$. There is therefore an element $\phi \in \mathscr{A}^{\wedge}$ such that kernel $\phi \supset I_{a}+[\zeta]$. Let $U$ be a neighborhood of $\phi$ in $\mathscr{A}^{\wedge}$. There is an ideal $I^{\prime}$ in $\mathscr{A}$ such that $U=\left\{\phi^{\prime} \in \mathscr{A}^{\wedge} \mid\right.$ kernel $\left.\phi^{\prime} \phi I^{\prime}\right\}$; so kernel $\phi \ngtr I^{\prime}$ and thus kernel $\left(\psi_{\zeta}\right)=[\zeta] \ngtr I^{\prime}$. This shows that $\left(\psi_{\zeta}\right) \in U$. So a net each of whose terms is $\left(\psi_{\xi}\right)$ converges to $\phi$ in $\mathscr{A}^{\wedge}$. But $\operatorname{Tr}(\phi(A))=0$ since 
$m(\mathscr{A})^{+} \subset I_{a}$. If $\sum A_{j} E_{j}$ is a spectral representation of $A$, then $\psi_{\zeta}(A)=\sum_{j} A_{j}(\zeta) E_{j}(\zeta)$ and so $\operatorname{Tr}\left(\psi_{\zeta}(A)\right)=\sum_{j} A_{j}(\zeta)<+\infty$. This shows that $\sum A_{j}(\zeta)=\operatorname{Tr}(\phi(A))=0$ and that $A(\zeta)=0$. We have therefore that $A(P-R)=0$ and consequently that $m(\mathscr{A})(P-R)=0$.

Since $I_{a}^{\wedge}$ is homeomorphic to the open subset $\left\{\phi \in \mathscr{A}^{\wedge} \mid\right.$ kernel $\left.\phi \ngtr I_{a}\right\}$ under inverse of the map $(\psi) \rightarrow\left(\psi \mid I_{a}\right)$, the function $\phi \rightarrow \operatorname{Tr}(\phi(A))$ is continuous on $I_{a}^{\wedge}$ for each $A \in m(\mathscr{A})^{+}$. This means that $m(\mathscr{A})^{+} \subset \mathscr{Q} R$. Let $A \in m(\mathscr{A})^{+}$. We show that $\lim _{n} \operatorname{Tr}(A) R_{n}=0$. Arguing by contradiction we assume that there is an $\varepsilon>0$ such that $\left\|\operatorname{Tr}(A) R_{n}\right\| \geqq \varepsilon$ for an infinite number of $n$. There is no loss in generality in assuming that $\left\|\operatorname{Tr}(A) R_{n}\right\| \geqq \varepsilon$ for every $n$, since we may replace $\left\{\operatorname{Tr}(A) R_{n}\right\}$ by a subsequence if necessary. For each $n$ there is a $\zeta_{n}$ in the set $Y_{n}=\left\{\zeta \in Z \mid R_{n}^{\wedge}(\zeta)=1\right\}$ such that $\left(\operatorname{Tr}(A) R_{n}\right)^{\wedge}\left(\zeta_{n}\right) \geqq \varepsilon / 2$. There is a cluster point $\zeta_{0}$ for the set $\left\{\zeta_{n} \mid n=1,2, \ldots\right\}$. We have that $\zeta_{0} \in Y-\bigcup_{n} Y_{n}$ where $Y=\left\{\zeta \in Z \mid R^{\wedge}(\zeta)=1\right\}$. However, $I_{a}+\left[\zeta_{0}\right]$ is a proper ideal in $\mathscr{A}$. Let $\phi \in \mathscr{A}^{\wedge}$ be such that kernel $\phi \supset I_{a}$ $+\left[\zeta_{0}\right]$. Every open neighborhood of $\phi$ in $\mathscr{A}^{\wedge}$ contains $\left(\psi_{\zeta_{0}}\right)$. Since $Z$ is homeomorphic to $I_{a}^{\wedge}=P_{a}$ and since $I_{a}^{\wedge}$ is an open subset of $\mathscr{A}^{\wedge},\left(\psi_{\xi_{0}}\right)$ is a cluster point of $\left\{\left(\psi_{\zeta_{n}}\right) \mid n=1,2, \ldots\right\}$ in $\mathscr{A}^{\wedge}$. So there is a subset of $\left\{\operatorname{Tr}\left(\psi_{\zeta_{n}}(A)\right)\right\}$ converging to $\operatorname{Tr}(\phi(A))$. This is impossible since $\operatorname{Tr}(\phi(A))=0$ and $\operatorname{Tr}\left(\psi_{\zeta_{n}}(A)\right) \geqq \varepsilon / 2$ for all $n$. Therefore, $\lim _{n} \operatorname{Tr}(A) R_{n}=0$.

Now suppose that $A \in \mathscr{Q} R$ and $\lim _{n} \operatorname{Tr}(A) R_{n}=0$. Since the set $\left\{\phi \in \mathscr{A}^{\wedge} \mid\right.$ kernel $\phi$ $\not \mathscr{A}(1-R)\}=\left\{\phi \in \mathscr{A}^{\wedge} \mid\right.$ kernel $\left.\phi \supset \mathscr{A} R\right\}$ is open and closed and since $\operatorname{Tr}(\phi(A))=0$ for all $\phi$ in this set, it is sufficient to prove that $\phi \rightarrow \operatorname{Tr}(\phi(A))$ is finite and continuous on $\left\{\phi \in \mathscr{A}^{\wedge} \mid\right.$ kernel $\left.\phi \supset \mathscr{A}(1-R)\right\}=\left\{\phi \in \mathscr{A}^{\wedge} \mid\right.$ kernel $\left.\phi \nsupseteq \mathscr{A} R\right\}=S$. Let $\phi_{0} \in S$; there is a unique $\zeta_{0} \in Y$ such that kernel $\phi_{0} \supset\left[\zeta_{0}\right]$. Let $\varepsilon>0$.

If $\zeta_{0} \in Y_{n}$ for some $n$, then $\left(\psi_{\zeta_{0}}\right)=\phi_{0}$. There is a projection $Q$ in $\mathscr{Z}$ such that $Q \leqq R_{n}$ such that $Q^{\wedge}\left(\zeta_{0}\right)=1$ and $\left|\operatorname{Tr}(A)^{\wedge}\left(\zeta_{0}\right)-\operatorname{Tr}(A)^{\wedge}(\zeta)\right|<\varepsilon$ whenever $Q^{\wedge}(\zeta)=1$. For every $\phi$ in the open set $\left\{\phi \in \mathscr{A}^{\wedge} \mid \operatorname{kernel} \phi \nsupseteq \mathscr{A} Q\right\}$, there is a $\zeta \in Z$ such that $Q^{\wedge}(\zeta)=1$ and $\left(\psi_{\zeta}\right)=\phi$. Indeed, there is a $\zeta \in Z$ such that kernel $\phi \supset[\zeta]$; so $\mathscr{A} Q$ is not

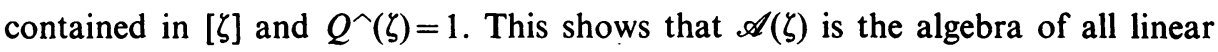
operators on an $n$-dimensional Hilbert space and so $\phi=\left(\psi_{\zeta}\right)$. So if $\phi \in \mathscr{A}^{\wedge}$ and kernel $\phi \ngtr \mathscr{A} Q$ then $\phi=\left(\psi_{\zeta}\right)$ for some $\zeta$ such that $Q^{\wedge}(\zeta)=1, \operatorname{Tr}(\phi(A))=\operatorname{Tr}(A)^{\wedge}(\zeta)$, and $\left|\operatorname{Tr}\left(\phi_{0}(A)\right)-\operatorname{Tr}(\phi(A))\right|=\left|\operatorname{Tr}(A)^{\wedge}\left(\zeta_{0}\right)-\operatorname{Tr}(A)^{\wedge}(\zeta)\right|<\varepsilon$.

If on the other hand $\zeta_{0} \in Y-\bigcup_{n} Y_{n}$ then $\operatorname{Tr}\left(\phi_{0}(A)\right)=0$ because $A\left(\zeta_{0}\right)=0$. There is an integer $N$ such that $\left\|\operatorname{Tr}(A)\left(1-\sum\left\{R_{n} \mid 1 \leqq n \leqq N\right\}\right)\right\|<\varepsilon$. The open set $V$ $=\left\{\phi \in \mathscr{A}^{\wedge} \mid\right.$ kernel $\left.\phi \not \mathscr{A}\left(1-\sum\left\{R_{n} \mid 1 \leqq n \leqq N\right\}\right)\right\}$ can be written as the union of three disjoint sets $S_{1}, S_{2}, S_{3}$ where $S_{1}=\left\{\phi \in \mathscr{A}^{\wedge} \mid\right.$ kernel $\left.\phi \not \mathscr{A}(1-R)\right\}, S_{2}=$ $\left\{\phi \in \mathscr{A}^{\wedge} \mid\right.$ kernel $\phi \supset[\zeta]$ for some $\zeta$ in $\left.Y-\bigcup_{n} Y_{n}\right\}$ and $S_{3}=\left\{\phi \in \mathscr{A}^{\wedge} \mid\right.$ kernel $\phi$

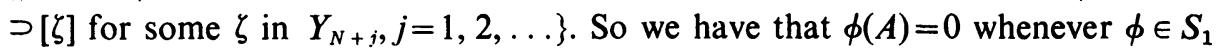
and $\phi(A)=0$ (because $A(\zeta)=0$ ) whenever $\phi \in S_{2}$. Finally, $\operatorname{Tr}(A)^{\wedge}(\zeta) \leqq \varepsilon$ whenever $\zeta \in Y_{N+j}(j=1,2, \ldots)$ and so $\operatorname{Tr}(\phi(A))=\operatorname{Tr}(A)^{\wedge}(\zeta) \leqq \varepsilon$ whenever $\phi \in S_{3}$ and kernel $\phi$ $\supset[\zeta]$. This shows that $\operatorname{Tr}(\phi(A)) \leqq \varepsilon$ on an open set $V$ containing $\phi_{0}$. Therefore, the function $\phi \rightarrow \operatorname{Tr}(\phi(A))$ is continuous on $\mathscr{A}^{\wedge}$. Q.E.D. 
4. The Schmidt class and the trace class of a Type I von Neumann algebra. Let $\mathscr{A}$ be a Type I von Neumann algebra with center $\mathscr{Z}$; let $I_{a}$ be the ideal generated by the abelian projections. Let $\mathscr{P}$ be the set of all positive elements $A$ in $I_{a}$ such that if $\sum A_{j} E_{j}$ is a spectral representation for $A$ then the increasing sequence $\left\{\sum\left(A_{j} \mid 1 \leqq j \leqq n\right)\right\}_{n}$ is strongly convergent in $\mathscr{Z}$. Thus, $A \in \mathscr{P}$ if and only if $\left\{\sum\left(A_{j} \mid 1 \leqq j \leqq n\right)\right\}$ is bounded above. The set $\mathscr{P}$ will serve as the starting point for the construction of the Schmidt class and the trace class.

If $A \in I_{a}^{+}$and if $\zeta \rightarrow \operatorname{Tr}(A(\zeta))$ is defined in a neighborhood of the point $\zeta_{0}$ in the spectrum of $\mathscr{Z}$ and is continuous at $\zeta_{0}$, then there is a central projection $P$ with $P^{\wedge}\left(\zeta_{0}\right)=1$ and $A P \in \mathscr{P}$. Indeed, if $\sum A_{j} E_{j}$ is a resolution of $A$ for all $\zeta$ in some open and closed neighborhood $U$ of $\zeta_{0}$ we have that $\operatorname{lub}_{n} \sum\left\{A_{\hat{j}}(\zeta) \mid 1 \leqq j \leqq n\right\} \leqq \operatorname{Tr}\left(A\left(\zeta_{0}\right)\right)$ +1 . Thus $\left\{\sum\left(A_{j} P \mid 1 \leqq j \leqq n\right)\right\}_{n}$ is bounded above in $\mathscr{Z}$. Here $P$ is the projection which corresponds to $U$.

The relation of $\mathscr{P}$ and $\mathscr{Q}(\S 3)$ is clarified by the following theorem based on a result of von Neumann [10].

TheORem 4.1. Let $A$ be an element of the set $\mathscr{P}$. There is a set $S$ of mutually orthogonal central projections of least upper bound 1 such that $P A \in \mathscr{Q}$ for each $P \in S$.

Proof. Let $\sum A_{j} E_{j}$ be a spectral resolution for $A$. It is sufficient to show that there is a nonzero projection $P$ in $\mathscr{Z}$ such that $\left\{\sum\left(A_{j} P \mid 1 \leqq j \leqq n\right)\right\}_{n}$ converges uniformly. Let $\mathscr{B}$ be the $C^{*}$-algebra generated by $\left\{A_{j} \mid j=1,2, \ldots\right\} \cup\{1\}$. The algebra $\mathscr{B}$ is separable. Let $H$ be the Hilbert space of $\mathscr{A}$ and let $x$ be a unit vector of $H$. The subspace $K=$ closure $\{B x \mid B \in \mathscr{B}\}$ is separable and the projection $E^{\prime}$ corresponding to $K$ is an element of the commutator $\mathscr{B}^{\prime}$ of $\mathscr{B}$ on $H$. If $E$ is the central support of $E^{\prime}$, then $E$ is an element of the commutative von Neumann algebra $\mathscr{B}$ " generated by $\mathscr{B}$ on $H$. Also $\mathscr{B}^{\prime \prime} \subset \mathscr{Z}$ because $\mathscr{B}^{\prime \prime}$ is the weak closure of $\mathscr{B}$ and $\mathscr{B}^{\prime \prime} E$ is isomorphic to the von Neumann algebra $\mathscr{B}^{\prime \prime} E^{\prime}$ on $E^{\prime}(H)$. Let $B_{n}=\sum\left\{A_{j} \mid 1 \leqq j \leqq n\right\} E^{\prime}, n=1$, $2, \ldots$ Then $\left\{B_{n}\right\}$ converges strongly to some $B$ in $\mathscr{B}^{\prime \prime} E^{\prime}$. There is a subsequence $\left\{B_{n_{k}}\right\}$ of $\left\{B_{n}\right\}$ and an element $C \in \mathscr{B}^{\prime \prime} E^{\prime}$ such that (1) $C y=0$ implies $y=0$ for $y \in E^{\prime}(H)$ and (2) $E^{\prime} \geqq \sum\left\{\left(\left(B_{n_{k}}-B\right) C\right)^{*}\left(\left(B_{n_{k}}-B\right) C\right) \mid 1 \leqq k \leqq m\right\}$ for all $m=1,2, \ldots$. So

$$
E^{\prime} \geqq C^{*} C \sum\left\{\left(B_{n_{k}}-B\right)^{*}\left(B_{n_{k}}-B\right) \mid 1 \leqq k \leqq m\right\}
$$

for all $m=1,2, \ldots$. There is a nonzero projection $F \in \mathscr{B}^{\prime \prime} E^{\prime}$ such that $F C^{*} C$ $\geqq 2^{-1} F\|C\|^{2}$. Therefore, there is an $\alpha>0$ such that

$$
\alpha F \geqq \sum\left\{\left(B_{n_{k}}-B\right)^{*}\left(B_{n_{k}}-B\right) \mid 1 \leqq k \leqq m\right\} F
$$

for all $m=1,2, \ldots$. For all $\zeta$ in the spectrum $X$ of $\mathscr{B}^{\prime \prime} E^{\prime}$ we have that

$$
\lim _{k} B_{n_{k}}^{\wedge}(\zeta) F^{\wedge}(\zeta)=B^{\wedge}(\zeta) F^{\wedge}(\zeta)
$$

Because $\left\{\left(B_{n_{k}} F\right)^{\wedge}\right\}_{k}$ is a monotonically increasing sequence of continuous functions on the compact set $X$, we have $\lim _{k} B_{n_{k}} F=B F$ (Dini's Theorem). So $\lim _{n} B_{n} F=B F$ 
(uniformly). Because $\mathscr{B}^{\prime \prime} E$ is isomorphic to $\mathscr{B}^{\prime \prime} E^{\prime}$ there is a projection $P$ in $\mathscr{Z}$ such that $\left\{\sum\left(A_{j} P \mid 1 \leqq j \leqq n\right)\right\}$ converges uniformly. Q.E.D.

Let $F$ be an abelian projection in the Type I algebra $\mathscr{A}$ with center $\mathscr{Z}$ and let $P$ be the central support of $F$. The algebra $F \mathscr{A} F$ is isomorphic to $\mathscr{Z} P$. We let $\tau_{F}(A)$ denote the unique element in $\mathscr{Z} P$ such that $F A F=\tau_{F}(A) F$.

We make use of the following lemma.

Lemma. Let $\mathscr{A}$ be a Type I von Neumann algebra and let $E$ and $F$ be abelian projections. Then $\tau_{E}(F)=\tau_{F}(E)$.

Proof. We have $F E F E F$ is equal to both $\tau_{E}(F) \tau_{F}(E) F$ and $\left(\tau_{F}(E)\right)^{2} F$; similarly, $\tau_{E}(F) \tau_{F}(E) E=\left(\tau_{E}(F)\right)^{2} E$. If $P$ (respectively $Q$ ) is the central support of $E$ (respectively $F$ ), the elements $\tau_{E}(F)$ and $\tau_{F}(E)$ are majorized by $P Q$. Let $\zeta$ be an element in the spectrum of the center of $\mathscr{A}$ such that $(P Q)^{\wedge}(\zeta)=1$. Then $F(\zeta) \neq 0$ and $E(\zeta) \neq 0$, and $\tau_{E}(F)^{\wedge}(\zeta)^{2}=\tau_{E}(F)^{\wedge}(\zeta) \tau_{F}(E)^{\wedge}(\zeta)=\tau_{F}(E)^{\wedge}(\zeta)^{2}$. So $\tau_{E}(F)^{\wedge}(\zeta)$ $=\tau_{F}(E)^{\wedge}(\zeta)$; this shows that $\tau_{E}(F)^{\wedge}(\zeta)=\tau_{E}(F)^{\wedge}(\zeta)$ for all $\zeta$ in the spectrum of the center. Thus, $\tau_{E}(F)=\tau_{F}(E)$.

Proposition 4.2. Let $\mathscr{A}$ be a Type I von Neumann algebra with center $\mathscr{Z}$ and let $I_{a}$ be the ideal generated by the abelian projections. Let $A$ be a positive element in $\mathscr{A}$, and let $S$ be a set of mutually orthogonal abelian projections. Let $F(S)$ be the set of all finite subsets of $S$. Let $N(A, S)$ be the increasing net in $\mathscr{Z}^{+}$given by

$$
\left\{\sum\left\{\tau_{F}(A) \mid F \in \pi\right\} \mid \pi \in F(S)\right\} .
$$

Then $A$ is an element of $\mathscr{P}$ if and only if $N(A, S)$ is bounded above for every set $S$ of mutually orthogonal abelian projections.

Proof. Let $A$ be an element of $\mathscr{P}$ and let $\sum A_{j} E_{j}$ be a spectral resolution of $A$. Let $\sum A_{j}$ denote the least upper bound of $\left\{\sum\left(A_{j} \mid 1 \leqq j \leqq n\right)\right\}_{n}$. Let $S$ be a set of mutually orthogonal abelian projections and let $F(S)$ be the set of all finite subsets of $S$. Then if $\pi \in F(S)$,

$$
\begin{aligned}
\sum\left\{\tau_{F}(A) \mid F \in \pi\right\} & =\sum\left\{\tau_{F}\left(\sum_{j} A_{j} E_{j}\right) \mid F \in \pi\right\} \\
& =\sum_{j} A_{j} \tau_{E_{j}}\left(\sum\{F \mid F \in \pi\}\right) \leqq A_{j}
\end{aligned}
$$

because $\lim _{n} \tau_{F}\left(\sum\left\{A_{j} E_{j} \mid 1 \leqq j \leqq n\right\}\right)=\tau_{F}(A)$ uniformly. Indeed, $\tau_{F}$ is an isometry of $F \mathscr{A} F$ onto $\mathscr{Z} P(P$ central support of $F)$ and $\lim _{n} \sum\left\{A_{j} E_{j} \mid 1 \leqq j \leqq n\right\}=A$ uniformly. This proves that $\left\{\sum\left\{\tau_{F}(A) \mid F \in \pi\right\} \mid \pi \in F(S)\right\}$ is bounded above by $\sum A_{j}$.

Conversely, suppose that $A$ is a positive element in $\mathscr{A}$ and that for every set $S$ of mutually orthogonal abelian projections the set $\left\{\sum\left\{\tau_{F}(A) \mid F \in \pi\right\} \mid \pi \in F(S)\right\}$ is bounded above. We show that $A$ is an element of $\mathscr{P}$. It is sufficient to show $A$ is an element of $I_{a}$. If $\varepsilon>0$ is given we find an element $B \in I_{a}$ such that $\|B-A\| \leqq \varepsilon$. Let $Z$ be the spectrum of the center of $\mathscr{A}$. Suppose $\zeta \in Z$; we show $A(\zeta) \in I_{a}(\zeta)$. Indeed, 
if $A(\zeta) \notin I_{a}(\zeta)$, there is a sequence $x_{j}$ of orthonormal vectors in $H(\zeta)$ such that the set $\left(\sum\left\{\left(A(\zeta) x_{j}, x_{j}\right) \mid 1 \leqq j \leqq n\right\}\right)_{n}$ is not bounded above because $I_{a}(\zeta)$ is the set of all completely continuous operators on $H(\zeta)$. Now there is a sequence $\left\{F_{j}\right\}$ of orthogonal abelian projections in $\mathscr{A}$ such that $F_{j}(\zeta)$ is the one-dimensional projection on the subspace generated by $x_{j}$. We have that $\left(\sum\left\{\tau_{F_{j}}(A) \mid 1 \leqq j \leqq n\right\}\right)_{n}$ is not bounded above in the center because $\left(\sum\left\{\tau_{F_{j}}(A) \mid 1 \leqq j \leqq n\right\}\right)^{\wedge}(\zeta)=\sum\left\{\left(A(\zeta) x_{j}, x_{j}\right) \mid 1 \leqq j \leqq n\right\}$ is not bounded above. This is a contradiction. So we conclude that $A(\zeta) \in I_{a}(\zeta)$ for every $\zeta$ in the spectrum of the center. Since $\zeta \rightarrow\|C(\zeta)\|(C \in \mathscr{A})$ is continuous on the spectrum $Z$ there are a finite number $\left\{U_{j} \mid 1 \leqq j \leqq m\right\}$ of disjoint open and closed sets and a corresponding number $\left\{B_{j} \mid 1 \leqq j \leqq m\right\}$ of elements in $I_{a}$ such that $\left\|A(\zeta)-B_{j}(\zeta)\right\|<\varepsilon$ for $\zeta \in U_{j}$ and $1 \leqq j \leqq m$. If $P_{j}(1 \leqq j \leqq m)$ is the projection of $\mathscr{Z}$ corresponding to $U_{j}(1 \leqq j \leqq m)$, then $B=\sum B_{j} P_{j} \in I_{a}$ and $\|A-B\| \leqq \varepsilon$. Q.E.D.

Let $A$ be an element of $\mathscr{P}$ and let $A$ have the spectral resolution $\sum A_{j} E_{j}$. Define the trace $\operatorname{Tr}(A)$ of $A$ to be the least upper bound of the increasing sequence $\left(\sum\left\{A_{j} \mid 1 \leqq j \leqq n\right\}\right)_{n}$ in $\mathscr{Z}$. The trace of $A$ is a function in $\mathscr{P}$ since the elements $A_{f}$ are uniquely determined by $A$. In fact we have the following proposition.

Proposition 4.3. Let $A$ be an element of the set $\mathscr{P}$. Let $S$ be a set of mutually orthogonal abelian projections in $\mathscr{A}$ of least upper bound 1 ; let $F(S)$ be the set of all finite subsets of $S$. Then

$$
\operatorname{lub}\left\{\sum\left\{\tau_{F}(A) \mid F \in \pi\right\} \mid \pi \in F(S)\right\}=\operatorname{Tr}(A) .
$$

Proof. From the proof of Proposition 4.2 we obtain that $\sum\left\{\tau_{F}(A) \mid F \in \pi\right\}$ $\leqq \operatorname{Tr}(A)$ for all $\pi \in F(S)$. So $B=\operatorname{lub}\left\{\sum\left\{\tau_{F}(A) \mid F \in \pi\right\} \mid \pi \in F(S)\right\} \leqq \operatorname{Tr}(A)$. Conversely, let $n$ be a positive integer. For every $\pi \in F(S)$ we have that

$$
\sum\left\{\tau_{F}\left(\sum\left\{A_{j} E_{j} \mid 1 \leqq j \leqq n\right\}\right) \mid F \in \pi\right\} \leqq B
$$

since $\sum\left\{A_{j} E_{j} \mid 1 \leqq j \leqq n\right\} \leqq A$. So $\sum\left\{A_{j} \tau_{E_{j}}\left(\sum\{F \mid F \in \pi\}\right) \mid 1 \leqq j \leqq n\right\} \leqq B$ for every $\pi \in F(S)$. However, for every abelian projection $E$, we have that

$$
\operatorname{lub}\left\{\tau_{E}\left(\sum\{F \mid F \in \pi\}\right) \mid \pi \in F(S)\right\}=1 .
$$

Indeed, $\lim _{\pi} \sum\{F \mid F \in \pi\}=1$ (strongly) and therefore $\lim _{\pi} \tau_{E}\left(\sum\{F \mid F \in \pi\}\right)=1$ (ultraweakly). Since $\left\{\tau_{E}\left(\sum\{F \mid F \in \pi\}\right) \mid \pi \in F(S)\right\}$ is bounded above, the strong limit of this increasing net exists and must be equal to the ultraweak limit 1 . This shows that

$$
\operatorname{lub}_{\pi} \sum\left\{A_{j} \tau_{E},\left(\sum\{F \mid F \in \pi\}\right) \mid 1 \leqq j \leqq n\right\}=\sum\left\{A_{j} \mid 1 \leqq j \leqq n\right\}
$$

is less than or equal to $B$. Because $n$ is arbitrary $\operatorname{Tr}(A) \leqq B$. Q.E.D.

Now we show that $\mathscr{P}$ is the set of positive elements of a two-sided ideal $T$ in $\mathscr{A}$.

THEOREM 4.4. Let $\mathscr{A}$ be a Type I von Neumann algebra and let $I_{a}$ be the ideal generated by the abelian projections of $\mathscr{A}$. Let $\mathscr{P}$ be the set of positive elements 
$A$ in $I_{a}$ such that if $\sum A_{j} E_{j}$ is a spectral representation of $A$ then $\left(\sum\left\{A_{j} \mid 1 \leqq j \leqq n\right\}\right)_{n}$ is bounded above. Let $\mathscr{S}=\left\{A \in \mathscr{A} \mid A^{*} A \in \mathscr{P}\right\}$; the set $\mathscr{S}$ is a two-sided ideal in $\mathscr{A}$.

Proof. It is sufficient to show that the following three facts about $\mathscr{P}$ are true: (1) $\mathscr{P}+\mathscr{P} \subset \mathscr{P}$; (2) if $A \in \mathscr{A}$ and $A^{*} A \in \mathscr{P}$, then $A A^{*} \in \mathscr{P}$; and (3) if $A \in \mathscr{A}, B \in \mathscr{P}$ and $0 \leqq A \leqq B$, then $A \in \mathscr{P}$.

First let $A$ and $B$ be elements of $\mathscr{P}$; then $A \in I_{a}^{+}$and $B \in I_{a}^{+}$. Let $S$ be a set of orthogonal abelian projections and let $F(S)$ be the set of all finite subsets of $S$. We have that $\sum\left\{\tau_{F}(A+B) \mid F \in \pi\right\}=\sum\left\{\tau_{F}(A) \mid F \in \pi\right\}+\sum\left\{\tau_{F}(B) \mid F \in \pi\right\} \leqq \operatorname{Tr}(A)$ $+\operatorname{Tr}(B)$. So $A+B \in \mathscr{P}$.

Now let $A \in \mathscr{A}$ and $A^{*} A \in \mathscr{P}$. Let $\sum A_{j} E_{j}$ be a spectral resolution of $A^{*} A$. Let $U$ be a partial isometric operator of $\mathscr{A}$ such that $U^{*} U$ is the domain support of $A$, $U U^{*}$ is the range support of $A$ and $U|A|=A\left(|A|=\left(A^{*} A\right)^{1 / 2}\right)$. We have that $\sum E$, is the domain support of $A^{*} A$ and thus $\sum E_{j}=U^{*} U$. The projection $F_{j}=U E_{j} U^{*}$ is abelian and equivalent to $E_{j}$. Also $A A^{*}=U|A||A| U^{*}=U\left(\sum A_{j} E_{j}\right) U^{*}=\sum A_{j} F_{j}$. However, $\sum A_{j} F_{j}$ is a spectral resolution for $A A^{*}$; thus, $A A^{*} \in \mathscr{P}$. Notice that $\operatorname{Tr}\left(A^{*} A\right)=\operatorname{Tr}\left(A A^{*}\right)$.

Finally, let $A \in \mathscr{A}, B \in \mathscr{P}$, and $0 \leqq A \leqq B$. Let $S$ be a set of mutually orthogonal abelian projections and let $F(S)$ be the set of all finite subsets of $S$. We have that $\sum\left\{\tau_{F}(A) \mid F \in \pi\right\} \leqq \sum\left\{\tau_{F}(B) \mid F \in \pi\right\} \leqq \operatorname{Tr}(B)$ for every $\pi \in F(S)$. This proves $A \in \mathscr{P}$. Q.E.D.

We define the Schmidt class of a Type I von Neumann algebra $\mathscr{A}$ to be the ideal $\mathscr{S}$ and the trace class of the algebra $\mathscr{A}$ to be the ideal $\mathscr{T}=\mathscr{S}^{2}$. The set of positive elements of $\mathscr{T}$ is then precisely the set $\mathscr{P}$.

The next proposition extends the trace $\operatorname{Tr}$ to $\mathscr{T}$.

Proposition 4.5. Let $\mathscr{A}$ be a Type I von Neumann algebra and let $\mathscr{T}$ be the trace class of $\mathscr{A}$. There is one and only one function $\operatorname{Tr}$ on $\mathscr{T}$ into the center $\mathscr{Z}$ of $\mathscr{A}$ with the following properties: (1) if $A$ is a positive element in $\mathscr{T}$ with spectral resolution $A=\sum A_{j} E_{j}$ then $\operatorname{Tr}(A)=\operatorname{lub}_{n} \sum\left\{A_{j} \mid 1 \leqq j \leqq n\right\} ;(2)$ if $A_{1}, A_{2}$ are elements of $\mathscr{T}$ and $C_{1}, C_{2}$ are elements of $\mathscr{Z}$ then $\operatorname{Tr}\left(C_{1} A_{1}+C_{2} A_{2}\right)=C_{1} \operatorname{Tr}\left(A_{1}\right)+C_{2} \operatorname{Tr}\left(A_{2}\right) ;$ (3) if $A \in \mathscr{T}$, then $\operatorname{Tr}\left(U^{*} A U\right)=\operatorname{Tr}(A)$ for every unitary operator $U$ in $\mathscr{A}$; and (4) if $A \in \mathscr{T}$ the function $f(B)=\operatorname{Tr}(B A)$ is continuous on $\mathscr{A}$.

Proof. Let $A_{1}$ and $A_{2}$ be elements of $\mathscr{P}$ and let $S$ be a set of mutually orthogonal abelian projections of least upper bound 1 ; let $F(S)$ be the set of all finite subsets of $S$. Suppose $\sum A_{k j} E_{k j}$ is a spectral resolution for $A_{k}(k=1,2)$. Let $n$ be given. Then

$$
\begin{aligned}
& \sum\left\{A_{1 j}+A_{2 j} \mid 1 \leqq j \leqq n\right\} \\
& \quad=\operatorname{lub}_{\pi} \sum\left[\tau_{F}\left(\sum\left\{A_{k j} E_{k j} \mid 1 \leqq j \leqq n ; k=1,2\right\}\right) \mid F \in \pi\right] \\
& \quad \leqq \operatorname{lub}_{\pi} \sum\left[\tau_{F}\left(A_{1}+A_{2}\right) \mid F \in \pi\right] \leqq \operatorname{Tr}\left(A_{1}\right)+\operatorname{Tr}\left(A_{2}\right) .
\end{aligned}
$$


Therefore, $\operatorname{Tr}\left(A_{1}\right)+\operatorname{Tr}\left(A_{2}\right) \leqq \operatorname{Tr}\left(A_{1}+A_{2}\right) \leqq \operatorname{Tr}\left(A_{1}\right)+\operatorname{Tr}\left(A_{2}\right)$. This proves that $\operatorname{Tr}\left(A_{1}+A_{2}\right)=\operatorname{Tr}\left(A_{1}\right)+\operatorname{Tr}\left(A_{2}\right)$ if $A_{1}, A_{2}$ are in $\mathscr{P}$.

Now let $C \in \mathscr{Z}^{+}$and let $A \in \mathscr{P}$. Assume $C \neq 0$. Let $P$ be the projection in $\mathscr{Z}$ corresponding to the closure of the open set of all $\zeta$ in the spectrum of $\mathscr{Z}$ such that $C^{\wedge}(\zeta) \neq 0$. Then $C A$ has the spectral resolution $\sum\left(C A_{j}\right)\left(E_{j} P\right)$ where $\sum A_{j} E_{j}$ is a spectral resolution of $A$. We have that

$$
\operatorname{Tr}(C A)=\operatorname{lub}_{n} \sum\left\{C A_{j} \mid 1 \leqq j \leqq n\right\}=C \operatorname{lub}_{n} \sum\left\{A_{j} \mid 1 \leqq j \leqq n\right\}=C \operatorname{Tr}(A) .
$$

If $A$ is a self-adjoint element in the trace class, then $A$ may be written as the difference $A=A_{1}-A_{2}$ of two elements $A_{1}$ and $A_{2}$ in $\mathscr{P}$. Setting $\operatorname{Tr}(A)=\operatorname{Tr}\left(A_{1}\right)$ $-\operatorname{Tr}\left(A_{2}\right)$ uniquely defines the function $\operatorname{Tr}$ on the self-adjoint elements of the trace class. If $A$ is an arbitrary element of the trace class, then $A$ may be written as a linear combination $A=A_{1}+i A_{2}$ of two self-adjoint elements $A_{1}$ and $A_{2}$ of the trace class. Setting $\operatorname{Tr}(A)=\operatorname{Tr}\left(A_{1}\right)+i \operatorname{Tr}\left(A_{2}\right)$ uniquely defines $\operatorname{Tr}$ on elements of the trace class. The function $\operatorname{Tr}$ will then have properties (1) and (2).

Now let $A \in \mathscr{P}$ and let $A=\sum A_{j} E_{j}$ be a resolution of $A$; let $U$ be a unitary element of $\mathscr{A}$. We see that $U^{*} A U \in \mathscr{P}$ and $\sum A_{j} U^{*} E_{j} U$ is a resolution for $U^{*} A U$. Thus, $\operatorname{Tr}(A)=\operatorname{Tr}\left(U^{*} A U\right)$. Since every $A$ in $\mathscr{T}$ is a linear combination of four elements in $\mathscr{P}, \operatorname{Tr}\left(U^{*} A U\right)=\operatorname{Tr}(A)$ for every $A$ in $\mathscr{P}$.

Finally, let $A \in \mathscr{T}^{+}$and let $B \in \mathscr{A}^{+}$. We have $\operatorname{Tr}\left(A^{1 / 2} B A^{1 / 2}\right) \leqq\|B\| \operatorname{Tr}(A)$ since $0 \leqq A^{1 / 2} B A^{1 / 2} \leqq\|B\| A$. So $\operatorname{Tr}(B A) \leqq\|B\| \operatorname{Tr}(A)$. If $B$ is an arbitrary element of $\mathscr{A}$, we may write $B=B_{1}-B_{2}+i\left(B_{3}-B_{4}\right)$ where $B_{j}$ is positive and $B_{1} B_{2}=B_{3} B_{4}=0$. Thus $\|\operatorname{Tr}(B A)\| \leqq \sum\left(\left\|B_{j}\right\|\|\operatorname{Tr}(A)\|\right) \leqq 4\|B\|\|\operatorname{Tr}(A)\|$. If $A$ is an arbitrary element of $\mathscr{T}$, we may write $A=A_{1}-A_{2}+i\left(A_{3}-A_{4}\right)$ where the $A_{j}$ are elements of $\mathscr{T}^{+}$. So $\|\operatorname{Tr}(B A)\| \leqq 4\|B\| \sum\left\|\operatorname{Tr}\left(A_{j}\right)\right\|$. This shows that the function $B \rightarrow \operatorname{Tr}(B A)$ is continuous on $\mathscr{A}$ for fixed $A$ in $\mathscr{T}$. Q.E.D.

Let $A$ and $B$ be two elements of the Schmidt class. Define the function of $\mathscr{S} \times \mathscr{S}$ into $\mathscr{Z}$ by $(A, B)=\operatorname{Tr}\left(B^{*} A\right)$. Then this function satisfies the properties: (1) $(A, B)$ $=(B, A)^{*} ;(2)(A, A) \geqq 0$ and $(A, A)=0$ if and only if $A=0$; and (3) $\left(C A+C^{\prime} A^{\prime}, B\right)$ $=C(A, B)+C^{\prime}\left(A^{\prime}, B\right)$ for $C, C^{\prime} \in \mathscr{Z}, A^{\prime} \in \mathscr{S}$. Define $|A|=(A, A)^{1 / 2}$ and $\|A\|_{1}=$ $\|(A, A)\|^{1 / 2}$. We have that $\|A\| \leqq\|A\|_{1}$ for all $A$ in $\mathscr{S}$. Indeed, let $\sum A_{j} E_{j}$ be a spectral resolution for $A^{*} A$. We have that $\left\|A_{1}\right\|=\left\|A^{*} A\right\|$. So $\|A\|^{2}=\left\|A^{*} A\right\|=\left\|A_{1}\right\|$ $\leqq\left\|\operatorname{Tr}\left(A^{*} A\right)\right\|=\|A\|_{1}^{2}$. The function $A \rightarrow\|A\|_{1}$ on $\mathscr{S}$ defines a norm [9]. We show that $\mathscr{S}$ is complete in this norm.

TheOREM 4.6. Let $\mathscr{A}$ be a Type I von Neumann algebra and let $\mathscr{S}$ be the Schmidt class of $\mathscr{A}$. Under the norm $\|A\|_{1}^{2}=\left\|\operatorname{Tr}\left(A^{*} A\right)\right\|$, the Schmidt class $\mathscr{S}$ is complete.

Proof. Let $\left\{A_{n}\right\}$ be a Cauchy sequence in $\mathscr{S}$. The relation $\|B\| \leqq\|B\|_{1}$ for $B \in \mathscr{S}$ implies that $\left\{A_{n}\right\}$ is a Cauchy sequence in $I_{a}$. There is an element $A$ in $I_{a}$ such that $\lim _{n}\left\|A_{n}-A\right\|=0$. We prove that $A \in \mathscr{S}$ and that $\lim _{n}\left\|A_{n}-A\right\|_{1}=0$. 
Let $S$ be a set of mutually orthogonal abelian projections and let $F(S)$ be the set of all finite subsets of $S$. For each $\pi \in F(S)$ we have that

$$
\begin{aligned}
& {\left[\sum\left\{\tau_{F}\left(\left(A-A_{n}\right)^{*}\left(A-A_{n}\right)\right) \mid F \in \pi\right\}\right]^{1 / 2} } \\
& \leqq {\left[\sum\left\{\tau_{F}\left(\left(A-A_{m}\right)^{*}\left(A-A_{m}\right)\right) \mid F \in \pi\right\}\right]^{1 / 2} } \\
&+\left[\sum\left\{\tau_{F}\left(\left(A_{m}-A_{n}\right)^{*}\left(A_{m}-A_{n}\right)\right) \mid F \in \pi\right\}\right]^{1 / 2}
\end{aligned}
$$

Indeed, let $\zeta$ be an arbitrary point in the spectrum of the center of $\mathscr{A}$. For each $F \in \pi, F(\zeta)$ is a projection on $H(\zeta)$ of dimension at most one. Let $x_{1}, x_{2}, \ldots, x_{n}$ be an orthonormal set of vectors in $H(\zeta)$ which respectively span the subspaces corresponding to the nonzero $F(\zeta)$ with $F \in \pi$. Then

$$
\begin{aligned}
& {\left[\sum\left\{\tau_{F}\left(\left(A-A_{n}\right)^{*}\left(A_{n}-A\right)\right) \mid F \in \pi\right\}\right]^{1 / 2}(\zeta) } \\
&=\left[\sum\left\|\left(A(\zeta)-A_{n}(\zeta)\right) x_{j}\right\|^{2}\right]^{1 / 2} \\
& \leqq {\left[\sum\left\|\left(A(\zeta)-A_{m}(\zeta)\right) x_{j}\right\|^{2}\right]^{1 / 2}+\left[\sum\left\|\left(A_{m}(\zeta)-A_{n}(\zeta)\right) x_{j}\right\|^{2}\right]^{1 / 2} } \\
&= {\left[\sum\left\{\tau_{F}\left(\left(A-A_{m}\right)^{*}\left(A-A_{m}\right)\right) \mid F \in \pi\right\}\right]^{1 / 2 \wedge}(\zeta) } \\
&+\left[\sum\left\{\tau_{F}\left(\left(A_{m}-A_{n}\right)^{*}\left(A_{m}-A_{n}\right)\right) \mid F \in \pi\right\}\right]^{1 / 2 \wedge}(\zeta) .
\end{aligned}
$$

Since $\zeta$ is arbitrary, we have the desired result. Therefore,

$$
\begin{aligned}
{\left[\sum\left\{\tau_{F}\left(\left(A-A_{n}\right)^{*}\left(A-A_{n}\right)\right) \mid F \in \pi\right\}\right]^{1 / 2} \leqq } & {\left.\left[\sum \tau_{F}\left(\left(A-A_{m}\right)^{*}\left(A-A_{m}\right)\right) \mid F \in \pi\right\}\right]^{1 / 2} } \\
& +\left(A_{n}-A_{m}, A_{n}-A_{m}\right)^{1 / 2}
\end{aligned}
$$

Since $\lim _{m}\left\|\tau_{F}\left(\left(A-A_{m}\right)^{*}\left(A-A_{m}\right)\right)\right\|=0$ and since $\left\{A_{n}\right\}$ is Cauchy, given any $\varepsilon>0$ there is an $N$ such that $n \geqq N$ implies $\left\|\left[\sum\left\{\tau_{F}\left(\left(A-A_{n}\right)^{*}\left(A-A_{n}\right)\right) \mid F \in \pi\right\}\right]^{1 / 2}\right\|<\varepsilon$, for every $\pi \in F(S)$. This shows that $A-A_{N} \in \mathscr{S}$ and thus that $A \in \mathscr{S}$. It also shows that $\left\|A-A_{n}\right\|_{1} \leqq \varepsilon$ whenever $n \geqq N$. Thus $\lim _{n}\left\|A-A_{n}\right\|_{1}=0$. We obtain that $\mathscr{S}$ is complete. Q.E.D.

Let $M$ be a module over a commutative $A W^{*}$-algebra $\mathscr{Z}$. Suppose there is an inner product $(A, B)$ of $M$ with values in $\mathscr{Z}$ satisfying (1) $(A, B)=(B, A)^{*} ;(2)$ $(C A, B)=C(A, B)$; (3) $\left(A+A^{\prime}, B\right)=(A, B)+\left(A^{\prime}, B\right) ; \quad$ and $(4) \quad(A, A) \geqq 0$ and $(A, A)=0$ if and only if $A=0$, for all $A, A^{\prime}, B$ in $M$ and $C$ in $\mathscr{Z}$. By setting $\|A\|_{1}$ $=\left\|(A, A)^{1 / 2}\right\|$ for each $A$ in $M$ we define a norm in $M$. The $\mathscr{Z}$-module $M$ is said to be an $A W^{*}$-module over $\mathscr{Z}$ if (1) $M$ is complete; (2) if $R$ is a set of mutually orthogonal projections in $\mathscr{Z}$ of least upper bound 1 and if $A \in M$, then $P A=0$ for every $P \in R$ implies $A=0$; and (3) if $R=\left\{P_{j} \mid j \in J\right\}$ is a set of mutually orthogonal projections in $\mathscr{Z}$ of least upper bound 1 and if $\left\{A_{j} \mid j \in J\right\}$ is a bounded set of elements of $M$, then there is an $A \in M$ such that $P_{j} A=P_{j} A_{j}$ for every $j \in J$ [9]. We show that the Schmidt class of a Type I von Neumann algebra $\mathscr{A}$ is an $A W^{*}$-module over the center. 
THEOREM 4.7. Let $\mathscr{A}$ be a Type I von Neumann algebra with center $\mathscr{Z}$. Let $\mathscr{S}$ be the Schmidt class of $\mathscr{A}$. Under the inner product $\operatorname{Tr}\left(B^{*} A\right)=(A, B)$ on $\mathscr{S}$, the ideal $\mathscr{S}$ is an $A W^{*}$-module over $\mathscr{Z}$.

Proof. The only point which remains to be verified is the following: if $\left\{P_{j} \mid j \in J\right\}$ is a set of mutually orthogonal central projections of least upper bound 1 and if $\left\{A_{j} \mid j \in J\right\}$ is a bounded set of elements in $\mathscr{S}$, there is an $A \in \mathscr{S}$ such that $A P_{j}$ $=A_{j} P_{j}$ for all $j$. However, let $\left\|\operatorname{Tr}\left(A_{j}^{*} A_{j}\right)\right\| \leqq n$ for all $j$; then $\left\|A_{j}\right\|^{2} \leqq\left\|\operatorname{Tr}\left(A_{j}^{*} A_{j}\right)\right\| \leqq n$. Let $A=\sum A_{j} P_{j}$ in $\mathscr{A}$. Let $S$ be a set of mutually orthogonal abelian projections. For each finite subset $\pi$ of $S$ and for each $j \in J$ we have

$$
P_{j}\left(\sum\left\{\tau_{F}\left(A^{*} A\right) \mid F \in \pi\right\}\right)=P_{j}\left(\sum\left\{\tau_{F}\left(A_{j}^{*} A_{j}\right) \mid F \in \pi\right\}\right) \leqq \operatorname{Tr}\left(A_{j}^{*} A_{j}\right) P_{j} \leqq n P_{j} .
$$

Thus,

$$
\sum\left\{\tau_{F}\left(A^{*} A\right) \mid F \in \pi\right\} \leqq n \cdot 1
$$

However, the set $\pi$ is arbitrary and $\left\{\sum\left\{\tau_{F}\left(A^{*} A\right) \mid F \in \pi\right\} \mid \pi\right.$ a finite subset of $\left.S\right\}$ is bounded above. Therefore $A \in \mathscr{S}$. So $\mathscr{S}$ is an $A W^{*}$-module over $\mathscr{Z}$.

Let $\mathscr{A}$ be a Type I von Neumann algebra with center $\mathscr{Z}$ and let $M$ be an $A W^{*}$ module over $\mathscr{Z}$. A function $\phi$ of $I_{a}$ (respectively, $M$ ) into $\mathscr{Z}$ is called a $\mathscr{Z}$-linear functional if $\phi\left(C_{1} A_{1}+C_{2} A_{2}\right)=C_{1} \phi\left(A_{1}\right)+C_{2} \phi\left(A_{2}\right)$ for all $C_{1}, C_{2} \in \mathscr{Z}$ and $A_{1}$, $A_{2} \in I_{a}$ (respectively, $A_{1}, A_{2} \in M$ ). We now show that the set of all uniformly continuous $\mathscr{Z}$-linear functionals on $I_{a}$ is identifiable with the trace class of $\mathscr{A}$. We need the following lemma to show this.

Lemma. Let $M$ be an $A W^{*}$-module over the $A W^{*}$-module $\mathscr{Z}$. If $\phi$ is a continuous $\mathscr{Z}$-linear functional on $M$, there is a unique $A_{0} \in M$ such that $\phi(A)=\left(A, A_{0}\right)$ for all $A \in M[9$, Theorem 5].

THEOREM 4.8. Let $\mathscr{A}$ be a Type I von Neumann algebra on the Hilbert space $H$ and let $\mathscr{Z}$ be the center of $\mathscr{A}$. Let $I_{a}$ be the ideal generated by the abelian projections of $\mathscr{A}$, let $\mathscr{T}$ be the trace class of $\mathscr{A}$ and let $\operatorname{Tr}$ denote the trace function on $\mathscr{T}$. If $\phi$ is a uniformly continuous $\mathscr{Z}$-linear functional of $I_{a}$ into $\mathscr{Z}$, there is a unique $A_{0}$ in $\mathscr{T}$ such that $\phi(A)=\operatorname{Tr}\left(A A_{0}\right)$ for all $A \in I_{a}$.

Proof. We first settle the question of the uniqueness of $A_{0}$. Suppose $\operatorname{Tr}\left(A A_{0}\right)$ $=\operatorname{Tr}\left(A A_{0}^{\prime}\right)$ for all $A \in I_{a}$. In particular if we let $A=\left(A_{0}-A_{0}^{\prime}\right)^{*}$, then $\left\|A_{0}-A_{0}^{\prime}\right\|_{1}=0$. So $A_{0}=A_{0}^{\prime}$.

The uniformly continuous $\mathscr{Z}$-linear functional $\phi$ is a linear combination of two uniformly continuous $\mathscr{Z}$-linear functionals $\phi_{1}(A)=\phi(A)+\phi\left(A^{*}\right)^{*}$ and $\phi_{2}(A)$ $=i\left(\phi(A)-\phi\left(A^{*}\right)^{*}\right)$. For each $A \in I_{a}$, we have that $\phi_{j}(A)=\phi_{j}\left(A^{*}\right)^{*}(j=1,2)$. So we may assume that $\phi(A)=\phi\left(A^{*}\right)^{*}$ for each $A \in I_{a}$.

Let $\psi$ be the restriction of $\phi$ to the Schmidt class $\mathscr{S}$. Because $\|A\| \leqq\|A\|_{1}$ for each $A \in \mathscr{S}, \psi$ is a $\mathscr{Z}$-linear functional on $\mathscr{S}$ which is continuous in the topology on $\mathscr{S}$ induced by $\|\cdot\|_{1}$. By the lemma, there is a unique $A_{0} \in \mathscr{S}$ such that $\psi(A)=\operatorname{Tr}\left(A_{0} A\right)$ 
for each $A \in \mathscr{S}$. For each abelian projection $E$ in $\mathscr{A}, \psi(E)=\psi\left(E^{*}\right)^{*}=\psi(E)^{*}$; therefore $\tau_{E}\left(A_{0}\right) \operatorname{Tr}(E)=\psi(E)$ is self-adjoint. Thus, $A_{0}$ is self-adjoint.

We prove that $A_{0}$ is an element of the trace class $\mathscr{T}$. In fact let $A_{1}$ and $A_{2}$ be the positive and negative parts of $A_{0}$ respectively. Let $P$ be the domain support of $A_{1}$ and let $S$ be a set of mutually orthogonal abelian projections majorized by $P$. Then for any finite subset $\pi$ of $S$ we have that

$$
\left\|\sum\left\{\tau_{F}\left(A_{0}\right) \mid F \in \pi\right\}\right\|=\left\|\operatorname{Tr}\left(\sum\{F \mid F \in \pi\} A_{0}\right)\right\|=\left\|\phi\left(\sum\{F \mid F \in \pi\}\right)\right\| \leqq\|\phi\| .
$$

So $\left\{\sum\left\{\tau_{F}\left(A_{0}\right) \mid F \in \pi\right\}\right\}_{\pi}$ is bounded above. This proves that $A_{1}=A_{0} P$ is an element of $\mathscr{T}$. Similarly $A_{2} \in \mathscr{T}$.

Finally the function $A \rightarrow \operatorname{Tr}\left(A A_{0}\right)$ is continuous on $\mathscr{A}$. The relation $\operatorname{Tr}\left(E A_{0}\right)$ $=\phi(E)$ for every abelian projection $E$ in $I_{a}$ implies that $\phi(A)=\operatorname{Tr}\left(A A_{0}\right)$ for all $A$ in $I_{a}$. Q.E.D.

Let $L\left(I_{a}, \mathscr{Z}\right)$ be the Banach space of all bounded linear transformations of $I_{a}$ into $\mathscr{Z}$. Let $L=L_{\mathscr{Z}}\left(I_{a}, \mathscr{Z}\right)$ be the set of all $\mathscr{Z}$-linear transformations in $L\left(I_{a}, \mathscr{Z}\right)$. The set $L$ is a linear manifold and is closed in the norm topology of $L\left(I_{a}, \mathscr{Z}\right)$. In fact if $\left\{\phi_{n}\right\}$ is a sequence in $L\left(I_{a}, \mathscr{Z}\right)$ which converges to $\phi$, then for each $C \in \mathscr{Z}$ and $A \in I_{a}$ we have $\phi(C A)=\lim _{n} \phi_{n}(C A)=C \lim \phi_{n}(A)=C \phi(A)$. This shows that $L$ is a Banach space under the norm induced on $L$ by $L\left(I_{a}, \mathscr{Z}\right)$.

THEOREM 4.9. Let $\mathscr{T}$ be the trace class of the Type I von Neumann algebra $\mathscr{A}$ with center $\mathscr{Z}$. For each $A \in \mathscr{T}$ let $\|A\|_{2}=\left\|\operatorname{Tr}\left(\left(A^{*} A\right)^{1 / 2}\right)\right\|$; the function $\|\cdot\|_{2}$ is a norm on $\mathscr{T}$ under which $\mathscr{T}$ is a Banach algebra with involution. If $L=L_{\mathscr{X}}\left(I_{a}, \mathscr{Z}\right)$ is the Banach space of all continuous $\mathscr{Z}$-linear functionals on $I_{a}$ and if $A_{\phi}$ is the unique element in $\mathscr{T}$ such that $\phi(A)=\operatorname{Tr}\left(A A_{\phi}\right)$ where $\phi \in L$, then the function $\phi \rightarrow A_{\phi}$ is an isometric isomorphism of $L$ onto $\mathscr{T}$ (as a Banach space).

Proof. Because the function $\phi \rightarrow A_{\phi}$ is an isomorphism, it is sufficient to prove $\|\phi\|=\left\|\operatorname{Tr}\left(\left(A_{\phi}^{*} A_{\phi}\right)^{1 / 2}\right)\right\|$ in order to show that $\mathscr{T}$ is a Banach space and that the function $\phi \rightarrow\left\|A_{\phi}\right\|$ is an isometry. Let $A^{\prime}=A_{\phi}$ and $\left|A^{\prime}\right|=\left(A^{\prime *} A^{\prime}\right)^{1 / 2}$. Let $U$ be a partial isometric operator in $\mathscr{A}$ such that $U\left|A^{\prime}\right|=A^{\prime}$. For each $A \in I_{a}$ we have

$$
\begin{aligned}
\|\phi(A)\| & =\left\|\operatorname{Tr}\left(A A^{\prime}\right)\right\| \leqq\left\|\operatorname{Tr}\left(\left(A U\left|A^{\prime}\right|^{1 / 2}\right)^{*}\left(A U\left|A^{\prime}\right|^{1 / 2}\right)\right)\right\|^{1 / 2}\left\|\operatorname{Tr}\left(\left|A^{\prime}\right|\right)\right\|^{1 / 2} \\
& \leqq\left\|U^{*} A^{*} A U\right\|^{1 / 2}\left\|\operatorname{Tr}\left(\left|A^{\prime}\right|\right)\right\| \leqq\|A\|\left\|\operatorname{Tr}\left(\left|A^{\prime}\right|\right)\right\| .
\end{aligned}
$$

Therefore, $\|\phi\| \leqq\left\|\operatorname{Tr}\left(\left|A^{\prime}\right|\right)\right\|$. Conversely, let $\sum A_{j} E_{j}$ be a spectral resolution for $\left|A^{\prime}\right|$. We have $\operatorname{lub}_{n}\left\|\sum\left\{A_{j} \mid 1 \leqq j \leqq n\right\}\right\|=\left\|\operatorname{Tr}\left(\left|A^{\prime}\right|\right)\right\|$. Because $F_{n}=\sum\left\{E_{j} \mid 1 \leqq j \leqq n\right\}$ is an element of $I_{a}$ of norm 1 , we obtain $\|\phi\| \geqq\left\|\operatorname{Tr}\left(F_{n} U^{*} A^{\prime}\right)\right\|=\left\|\operatorname{Tr}\left(F_{n}\left|A^{\prime}\right|\right)\right\|$ $=\left\|\sum\left\{A_{j} \mid 1 \leqq j \leqq n\right\}\right\|$. Hence $\|\phi\| \geqq\left\|\operatorname{Tr}\left(\left|A^{\prime}\right|\right)\right\|$. This shows $\|\phi\|=\left\|\operatorname{Tr}\left(\left|A^{\prime}\right|\right)\right\|$.

Because $\|A\|=\||A|\| \leqq\|\operatorname{Tr}(|A|)\|$ for every $A \in \mathscr{T}$, we have $\|A B\|_{2} \leqq\|A\|\|B\|_{2}$ $\leqq\|A\|_{2}\|B\|_{2}$ for every $A$ and $B$ in $\mathscr{T}$. So $\mathscr{T}$ is a Banach algebra.

Finally, let $A \in \mathscr{T}$ and let $U|A|=A$ be the polar decomposition of $A$. Then $\left|A^{*}\right|=U|A| U^{*}$. Therefore, $\left\|A^{*}\right\|_{2}=\operatorname{Tr}\left(U|A| U^{*}\right)=\operatorname{Tr}(|A|)=\|A\|_{2}$. Therefore, $\mathscr{T}$ is a Banach *-algebra. Q.E.D. 
THEOREM 4.10. Let $\mathscr{A}$ be a Type I von Neumann algebra with center $\mathscr{Z}$ over the Hilbert space $H$ and let $\mathscr{T}$ be the trace class considered as a Banach space with that topology induced by the norm $\|\cdot\|_{2}$. Let $A_{0} \in \mathscr{A}$; the function $\phi(A)=\operatorname{Tr}\left(A A_{0}\right)$ is a continuous $\mathscr{Z}$-linear function of $\mathscr{T}$ to $\mathscr{Z}$. Conversely, if $\phi$ is a continuous $\mathscr{Z}$-linear function of $\mathscr{T}$ into $\mathscr{Z}$, there is a unique $A_{\phi} \in \mathscr{A}$ such that $\phi(A)=\operatorname{Tr}\left(A A_{\phi}\right)$ for all $A \in \mathscr{T}$. The function $\phi \rightarrow A_{\phi}$ is an isometric isomorphism of the Banach space of all continuous $\mathscr{Z}$-linear functions of $\mathscr{T}$ into $\mathscr{Z}$ onto $\mathscr{A}$.

Proof. Let $A_{0} \in \mathscr{A}$ and let $A \in \mathscr{T}$. Then we have

$$
\begin{aligned}
\left\|\operatorname{Tr}\left(A A_{0}\right)\right\| & =\left\|\operatorname{Tr}\left(U|A| A_{0}\right)\right\|=\left\|\operatorname{Tr}\left(|A|^{1 / 2}|A|^{1 / 2} A_{0} U\right)\right\| \\
& \leqq\left\|\operatorname{Tr}(|A|)^{1 / 2}\right\|\left\|\operatorname{Tr}\left(U^{*} A_{0}^{*}|A| A_{0} U\right)^{1 / 2}\right\| \\
& \leqq\left\|\operatorname{Tr}(|A|)^{1 / 2}\right\|^{2}\left\|A_{0} U U^{*} A_{0}^{*}\right\|^{1 / 2} \leqq\left\|A_{0}\right\|\|\operatorname{Tr}(|A|)\|,
\end{aligned}
$$

where $U|A|$ is the polar decomposition of $A$. Thus $\phi(A)=\operatorname{Tr}\left(A A_{0}\right)$ is a continuous $\mathscr{Z}$-linear function on $\mathscr{T}$ and $\|\phi\| \leqq\left\|A_{0}\right\|$. On the other hand, given $\varepsilon>0$ there are two abelian projections $E$ and $F$ both having central support 1 such that $\left\|E A_{0} F\right\|$ $\geqq\left\|A_{0}\right\|-\varepsilon$. There is a partial isometric operator $U$ in $\mathscr{T}$ such that $U^{*} U=E$ and $U U^{*}=F$. Because $\operatorname{Tr}(|U|)=\operatorname{Tr}(E)=1$, we have that

$$
\begin{aligned}
\|\phi\| \geqq\left\|\operatorname{Tr}\left(U A_{0}\right)\right\| & =\left\|\operatorname{Tr}\left(\tau_{F}\left(U A_{0}\right) F\right)\right\| \\
& =\left\|\tau_{F}\left(U A_{0}\right)\right\|=\left\|F A_{0}^{*} U^{*} U A_{0} F\right\|^{1 / 2}=\left\|E A_{0} F\right\| \geqq\left\|A_{0}\right\|-\varepsilon .
\end{aligned}
$$

Since $\varepsilon>0$ is arbitrary, we have $\|\phi\| \geqq\left\|A_{0}\right\|$. Hence, we have proved that $\|\phi\|=\left\|A_{0}\right\|$.

Let $\phi$ be a continuous $\mathscr{Z}$-linear functional of $\mathscr{T}$ into $\mathscr{Z}$. Let $\mathscr{S}$ be the Schmidt class of $\mathscr{A}$ with the norm $\|A\|_{1}=\left\|\operatorname{Tr}\left(A^{*} A\right)^{1 / 2}\right\|$. For every fixed $B \in \mathscr{S}$ the function $A \rightarrow \phi\left(B^{*} A\right)$ on $\mathscr{S}$ is a continuous $\mathscr{Z}$-linear functional. In fact, $\left\|\phi\left(B^{*} A\right)\right\| \leqq$ $\|\phi\|\left\|\operatorname{Tr}\left(\left|B^{*} A\right|\right)\right\|$. Now we obtain an estimate for $\left\|\operatorname{Tr}\left(\left|B^{*} A\right|\right)\right\|[12$, Lemma 5.14]. Let $A=U|A|$ be the polar decomposition of $A$ and let $B^{*} A=V\left|B^{*} A\right|$ be the polar decomposition of $B^{*} A$. Then $\left|B^{*} A\right|=V^{*} B^{*} A=V^{*} B^{*} U|A|$. Because $V^{*} B^{*} U$ and $|A|$ are elements of $\mathscr{S}$ we have

$$
\begin{aligned}
\left\|\operatorname{Tr}\left(\left|B^{*} A\right|\right)\right\| & \leqq\left\|\operatorname{Tr}\left(\left(V^{*} B^{*} U\right)^{*}\left(V^{*} B^{*} U\right)\right)^{1 / 2}\right\| \cdot\left\|\operatorname{Tr}\left(|A|^{2}\right)^{1 / 2}\right\| \\
& =\left\|\operatorname{Tr}\left(U^{*} B V^{*} V B^{*} U\right)^{1 / 2}\right\|\left\|\operatorname{Tr}\left(|A|^{2}\right)^{1 / 2}\right\| \\
& \leqq\|\| U\|\| V\left\|\operatorname{Tr}\left(B^{*} B\right)^{1 / 2}\right\|\left\|\operatorname{Tr}\left(A^{*} A\right)^{1 / 2}\right\| \leqq\|B\|_{1}\|A\|_{1} .
\end{aligned}
$$

Therefore $\left\|\phi\left(B^{*} A\right)\right\| \leqq\|\phi\|\|B\|_{1}\|A\|_{1}$.

There is for every $B \in \mathscr{S}$ a unique $\Phi(B) \in \mathscr{S}$ such that $\phi\left(B^{*} A\right)=\operatorname{Tr}\left(\Phi(B)^{*} A\right)$ $=(A, \Phi(B))$ for every $A \in \mathscr{S}$. Let $F$ be a projection in $I_{a}$. Define $\theta(F)=F \Phi(F) F$. Let $E$ be an abelian projection. Then

$$
\begin{aligned}
\left\|\tau_{E}(\Phi(F))\right\| & =\|(E, \Phi(F))\|=\|\phi(F E)\| \leqq\|\phi\|\|\operatorname{Tr}(|F E|)\| \\
& =\|\phi\|\left\|\operatorname{Tr}\left((E F F E)^{1 / 2}\right)\right\|=\|\phi\|\left\|\operatorname{Tr}\left(\left(\tau_{E}(F)\right)^{1 / 2} E\right)\right\| \\
& =\|\phi\|\left\|\tau_{E}(F)^{1 / 2}\right\| \leqq\|\phi\| .
\end{aligned}
$$

Therefore, $\|\Phi(F)\| \leqq 2\|\phi\|$ and $\|\theta(F)\| \leqq 2\|\phi\|$. 
Let $S$ be the set of all projections in $I_{a}$; the least upper bound (in the lattice of projections of $\mathscr{A}$ ) of two projections of $I_{a}$ is again a projection in $I_{a}$. Therefore, $S$ is a directed set under the usual ordering for projections.

If $F$ and $G$ are two projections in $S$ such that $F \leqq G$, then $\theta(F)=F \theta(G) F$. Indeed, for every $A \in \mathscr{S}$ we have $(A, \theta(F))=(F A F, \Phi(F))=\phi(F A F)=\phi(G F A F G)$ $=(G F A F G, \Phi(G))=(A, F \theta(G) F)$. Hence, if $x \in H$ and if there is a projection $F \in S$ such that $F x=x$, then the net $\{(\theta(E) x, x) \mid E \in S\}$ is eventually constant. So for every such $x \in H, \lim _{S}(\theta(E) x, x)$ exists. Let $x$ be an arbitrary element in $H$. We show that $\lim _{S}(\theta(E) x, x)$ exists by showing that the net $\{(\theta(E) x, x) \mid E \in S\}$ is Cauchy. Let $\varepsilon>0$ be given. Let $G$ be an element in $S$ such that $\|(1-G) x\|<\varepsilon$ and let $y=G x$. For every $F$ and $F^{\prime}$ in $S$ such that $F \geqq G$ and $F^{\prime} \geqq G$ we have $(\theta(F) y, y)=\left(\theta\left(F^{\prime}\right) y, y\right)$. Thus,

$$
\begin{aligned}
\left|(\theta(F) x, x)-\left(\theta\left(F^{\prime}\right) x, x\right)\right| \leqq & |(\theta(F) x, x)-(\theta(F) y, y)| \\
& +\left|\left(\theta\left(F^{\prime}\right) y, y\right)-\left(\theta\left(F^{\prime}\right) x, x\right)\right| \leqq|(\theta(F)(x-y), x)| \\
& +|(\theta(F) y, x-y)|+\left|\left(\theta\left(F^{\prime}\right)(y-x), y\right)\right| \\
& +\left|\left(\theta\left(F^{\prime}\right) x, y-x\right)\right| \leqq 8 \varepsilon\|\phi\|\|x\|
\end{aligned}
$$

because $\|\theta(E)\| \leqq 2\|\phi\|$ for all $E \in S$. This shows that for every $x \in H$ the net $\{(\theta(E) x, x) \mid E \in S\}$ converges. By the standard arguments there is a bounded linear operator $A_{0}$ on $H$ such that (1) $\left\|A_{0}\right\| \leqq 2\|\phi\|$ and (2) $\lim _{S}(\theta(E) x, y)=\left(A_{0} x, y\right)$ for every $x, y \in H$. Therefore, $A_{0}$ is an element of $\mathscr{A}$.

Now let $E$ be a projection in the set $S$. We have $\left(E A_{0} E x, y\right)=\lim _{S}(\theta(F) E x, E y)$ $=(\theta(E) x, y)$. This means that $E A_{0} E=\theta(E)$.

Let $B \in \mathscr{S}$ and $C \in \mathscr{S}$ and let $E$ be a projection in $I_{a}$. We have that the range projection of $B^{*} E C E$ is equivalent to a projection majorized by $E$. So the range projection of $B^{*} E C E$ is in the set $S$. Let $F$ be a projection in $S$ majorizing both $E$ and the range projection of $B^{*} E C E$. Then

$$
\begin{aligned}
\left(C, E B A_{0} E\right) & =\left(E C E, B A_{0}\right)=\left(B^{*} E C E, A_{0} E\right)=\left(F B^{*} E C E F, A_{0} E\right) \\
& =\left(B^{*} E C E, F A_{0} F\right)=\left(B^{*} E C E, \theta(F)\right)=\left(B^{*} E C E, \Phi(F)\right) \\
& =\phi\left(F B^{*} E C E\right)=\phi\left(B^{*} E C E\right)=(E C E, \Phi(B))=(C, E \Phi(B) E) .
\end{aligned}
$$

So for every $E \in S, E \Phi(B) E=E B A_{0} E$. This proves that $\Phi(B)=B A_{0}$.

Finally, let $B \in \mathscr{P}$. Then $B^{1 / 2} \in \mathscr{S}$. We have

$$
\phi(B)=\phi\left(B^{1 / 2} B^{1 / 2}\right)=\left(B^{1 / 2}, \Phi\left(B^{1 / 2}\right)\right)=\left(B^{1 / 2}, B^{1 / 2} A_{0}\right)=\operatorname{Tr}\left(A_{0}^{*} B\right) .
$$

Therefore, for every $B \in \mathscr{T}, \phi(B)=\operatorname{Tr}\left(A_{0}^{*} B\right)$.

If $\operatorname{Tr}\left(B A_{0}\right)=\operatorname{Tr}\left(B A_{0}^{\prime}\right)$ for every $B \in \mathscr{T}$, then $\tau_{E}\left(A_{0}-A_{0}^{\prime}\right)$ for every abelian projection. This shows that $A_{0}=A_{0}^{\prime}$. Therefore, if $\phi$ is a continuous $\mathscr{Z}$-linear function of function $\mathscr{T}$ into $\mathscr{Z}$ there is one and only one $A_{\phi}$ in $\mathscr{A}$ such that $\phi(A)$ $=\operatorname{Tr}\left(A A_{\phi}\right)$ for all $A \in \mathscr{T}$. Since the function $\phi \rightarrow A_{\phi}$ is linear (in fact it is $\mathscr{Z}$-linear), 
the function is an isometric isomorphism of the Banach space of all continuous $\mathscr{Z}$-linear functions of $\mathscr{T}$ into $\mathscr{Z}$ onto $\mathscr{A}$. Q.E.D.

We now study the properties of the trace Tr relative to the weak topology. If $\mathscr{A}$ and $\mathscr{B}$ are two von Neumann algebras, a linear function $\Phi$ of $\mathscr{A}$ into $\mathscr{B}$ is said to be normal if (1) $\Phi\left(\mathscr{A}^{+}\right) \subset \mathscr{B}^{+}$and (2) if $\left\{A_{n}\right\}$ is a monotonically increasing net in $\mathscr{A}^{+}$ such that $\operatorname{lub}_{n} A_{n}=A\left(A \in \mathscr{A}^{+}\right)$then $\operatorname{lub}_{n} \Phi\left(A_{n}\right)=\Phi(A)$. A linear function $\Phi$ of $\mathscr{A}$ into $\mathscr{B}$ which carries $\mathscr{A}^{+}$into $\mathscr{B}^{+}$is normal if and only if $\Phi$ is continuous in the ultraweak topologies of $\mathscr{A}$ and $\mathscr{B}$. The ultraweak topology for $\mathscr{A}$ on the Hilbert space $H$ is the weakest topology such that all linear functionals of the form $A \rightarrow \sum\left\{\left(A x_{j}, y_{j}\right) \mid 1 \leqq j<\infty\right\}$ are continuous where $\left\{x_{j}\right\}$ and $\left\{y_{j}\right\}$ are sequences of $H$ such that $\sum\left\|x_{j}\right\|^{2}<+\infty$ and $\sum\left\|y_{j}\right\|^{2}<+\infty$.

If $A$ is an element of the trace class the function $B \rightarrow \operatorname{Tr}(B A)$ is an ultraweakly continuous linear function of $\mathscr{A}$ into its center. We prove this in the following form.

THEOREM 4.11. Let $\mathscr{A}$ be a Type I von Neumann algebra with center $\mathscr{Z}$ and let $\mathscr{T}$ be the trace class of $\mathscr{A}$. Let $\mathscr{P}$ be the set of positive elements of $\mathscr{T}$. Assume that $\left\{A_{n} \mid n \in D\right\}$ is a monotonically increasing net of elements of $\mathscr{P}$ which is bounded above and suppose $A=\operatorname{lub}_{n} A_{n}$ is an element of $\mathscr{P}$. Then lub $\operatorname{Tr}\left(A_{n}\right)=\operatorname{Tr}(A)$.

Proof. If $x$ is an arbitrary vector of the Hilbert space of $\mathscr{A}$, it is sufficient to show $\lim _{n} \operatorname{Tr}\left(A_{n}\right) x=\operatorname{Tr}(A) x$ in order to show $\operatorname{lub}_{n} \operatorname{Tr}\left(A_{n}\right)=\operatorname{Tr}(A)$ because $\left\{\operatorname{Tr}\left(A_{n}\right) \mid n \in D\right\}$ is a monotonically increasing net in $\mathscr{Z}$ bounded above by $\operatorname{Tr}(A)$. Let $S$ be a set of mutually orthogonal abelian projections of least upper bound 1 and let $F(S)$ be the collection of finite subsets of $S$. For each $E \in S$ we have $\tau_{E}\left(A_{n}\right)$ $\leqq \tau_{E}(A)$ and $\lim _{n} \tau_{E}\left(A_{n}\right)=\tau_{E}(A)$ (strongly). Now given $\varepsilon>0$ there is an element $\pi$ in $F(S)$ such that $\left\|\sum\left\{\tau_{F}(A) \mid F \in \pi^{\prime}\right\} x\right\|<\varepsilon$ for every $\pi^{\prime} \in F(S)$ such that $\pi^{\prime} \cap \pi=\varnothing$. So $\left\|\sum\left\{\tau_{F}\left(A_{n}\right) \mid F \in \pi^{\prime}\right\} x\right\|<\varepsilon$ for every $\pi^{\prime} \in F(S)$ such that $\pi^{\prime} \cap \pi=\varnothing$ and for every $n \in D$. There is an $n_{0} \in D$ such that $\left\|\left(\sum\left\{\tau_{F}\left(A_{n}\right) \mid F \in \pi\right\}-\sum\left\{\tau_{F}(A) \mid F \in \pi\right\}\right) x\right\|<\varepsilon$ whenever $n \geqq n_{0}$. Therefore, if $\pi^{\prime}$ is in $F(S)$ and $\pi^{\prime} \supset \pi$ we have

$$
\left\|\left(\sum\left\{\tau_{F}\left(A_{n}\right) \mid F \in \pi^{\prime}\right\}-\sum\left\{\tau_{F}(A) \mid F \in \pi^{\prime}\right\}\right) x\right\|<3 \varepsilon,
$$

whenever $n \geqq n_{0}$. Thus, $\left\|\left(\operatorname{Tr}\left(A_{n}\right)-\operatorname{Tr}(A)\right) x\right\| \leqq 3 \varepsilon$ whenever $n \geqq n_{0}$. This proves that $\lim _{n} \operatorname{Tr}\left(A_{n}\right) x=\operatorname{Tr}(A) x$ and that $\lim _{n} \operatorname{Tr}\left(A_{n}\right)=\operatorname{Tr}(A)$ (strongly). Q.E.D.

COROllary. Let $A$ be an element of the trace class. The linear function $\phi(B)$ $=\operatorname{Tr}(B A)$ of $\mathscr{A}$ into $\mathscr{Z}$ is ultraweakly continuous.

Proof. We may write $A$ as a linear combination of four positive elements in the trace class. So we may assume that $A$ is an element of $\mathscr{P}$. If $B \in \mathscr{A}^{+}$then $\phi(B)$ $=\operatorname{Tr}(B A)=\operatorname{Tr}\left(B^{1 / 2} A B^{1 / 2}\right) \geqq 0$; therefore $\phi$ is a positive function. If $\left\{B_{n} \mid n \in D\right\}$ is a monotonically increasing net in $\mathscr{A}^{+}$with least upper bound $B$, then $\left\{A^{1 / 2} B_{n} A^{1 / 2} \mid n \in D\right\}$ is a monotonically increasing net in the trace class with least upper bound $A^{1 / 2} B A^{1 / 2}$ in the trace class. This means that $\operatorname{lub}_{n} \phi\left(B_{n}\right)=$ 
$\operatorname{lub}_{n} \operatorname{Tr}\left(A^{1 / 2} B_{n} A^{1 / 2}\right)=\operatorname{Tr}\left(A^{1 / 2} B A^{1 / 2}\right)=\phi(B)$. This shows that $\phi$ is ultraweakly continuous. Q.E.D.

We now extend the trace $\operatorname{Tr}$ to $\mathscr{A}^{+}$. Let $\left\{P_{j} \mid j \in J\right\}$ be a set of mutually orthogonal central projections such that for each $j \mathscr{Z}_{j}=\mathscr{Z} P_{j}$ is $\sigma$-finite. If $H$ is the Hilbert space of $\mathscr{A}$, let $x_{j}$ be a separating vector in $P_{j} H$ for $\mathscr{Z}_{j}$. Let $Z_{j}$ be the spectrum of $\mathscr{Z}_{j}$. This is identified with the compact set $\left\{\zeta \in Z \mid P_{j}^{\wedge}(\zeta)=1\right\}$. Let $\nu_{j}$ be the measure on $Z_{j}$ such that $w_{j}(A)=\left(A x_{j}, x_{j}\right)=\int_{Z_{j}} A^{\wedge}(\zeta) d v_{j}(\zeta)$ for every $A \in \mathscr{Z}_{j}$. Let $X=\bigcup\left\{Z_{j} \mid j \in J\right\}$ with the topology induced by the union. Let $\nu$ be the measure on $X$ induced by each $v_{j}$ acting on $Z_{j}$. Then $\mathscr{Z}$ is isomorphic isometric to the algebra $L_{\boldsymbol{C}}^{\infty}(X, v)$ of all essentially bounded complex-valued functions on $X$.

For each $j$ the function $w_{j} \cdot \operatorname{Tr}(A)$ on $\mathscr{P P} P_{j}$ is normal in the sense of Theorem 4.11. There is a unique faithful normal semifinite trace $\phi_{j}$ on $\mathscr{A}^{+} P_{j}$ such that $\phi_{j}(A)$ $=w_{j} \cdot \operatorname{Tr}(A)$ for every $A \in \mathscr{P P} P_{j}$. If $F(J)$ denotes the collection of all finite subsets of $J$, the function of $\mathscr{A}^{+}$defined by

$$
\phi(A)=\sup \left\{\sum\left(\phi_{j}(A) \mid j \in \pi\right) \mid \pi \in F(J)\right\}
$$

defines a faithful normal semifinite trace on $\mathscr{A}^{+}$. Let $\mathscr{Z}^{\sim+}$ be the set of all positive (finite or infinite) $v$-measurable functions on $X$. Define the trace $w$ on $\mathscr{Z}^{\sim+}$ by $w(f)=\sup$ \{outer integral of $f g_{Y} \mid g_{Y}$ characteristic function of a compact set $Y$ \}. There is a faithful normal \#-map $\Phi$ of $\mathscr{A}^{+}$into $\mathscr{Z}^{\sim+}$ such that $w \cdot \Phi(A)=\phi(A)$ for all $A \in \mathscr{A}^{+}$. If $A \in \mathscr{P} P_{j}$, then $w_{j}\left(\operatorname{Tr}\left(A P_{j}\right)\right)=\phi(A)=w \Phi(A)=w_{j}\left(\Phi\left(A P_{j}\right)\right)$. However, if $B$ is any element in $\mathscr{Z}_{j}^{+}$then $w_{j}(B \operatorname{Tr}(A))=w_{j}(B \Phi(A))$; so $\operatorname{Tr}(A)$ $=\Phi(A)$.

For any $A \in \mathscr{P}, \operatorname{Tr}(A) P_{j}=\Phi(A) P_{j}$ for all $j$. Therefore, $\Phi(A) \in \mathscr{Z}$ and $\operatorname{Tr}(A)$ $=\Phi(A)$. [2, III, §1, (Problem 11) and \$4; I, §6 (Proposition 9)].

Let $\phi$ be an ultraweakly continuous $\mathscr{Z}$-linear function of $\mathscr{A}$ into $\mathscr{Z}$. The functions $\phi_{1}(A)=2^{-1}\left(\phi(A)+\phi\left(A^{*}\right)^{*}\right)$ and $\phi_{2}(A)=(2 i)^{-1}\left(\phi(A)-\phi\left(A^{*}\right)^{*}\right)$ are $\mathscr{Z}$-linear ultraweakly continuous functions such that $\phi_{1}\left(A^{*}\right)=\phi_{1}(A)^{*}$ and $\phi_{2}\left(A^{*}\right)=\phi_{2}(A)^{*}$. We say that a $\mathscr{Z}$-linear function $\phi$ on $\mathscr{A}$ is hermitian if $\phi\left(A^{*}\right)=\phi(A)^{*}$ for each $A$ in $\mathscr{A}$. Thus, every ultraweakly continuous $\mathscr{Z}$-linear function may be written as a linear combination of two hermitian ultraweakly continuous $\mathscr{Z}$-linear functions.

The next theorem shows that each ultraweakly continuous hermitian $\mathscr{Z}$-linear function may be written as the difference of normal $\mathscr{Z}$-linear functions.

TheOREM 4.12. Let $\mathscr{A}$ be a Type I von Neumann algebra with center $\mathscr{Z}$ on the Hilbert space $H$. Let $\phi$ be a hermitian ultraweakly continuous $\mathscr{Z}$-linear function of $\mathscr{A}$ into $\mathscr{Z}$. There is a projection $E$ in $\mathscr{A}$ such that $\phi(A E)=\phi(E A)$ for every $A$ in $\mathscr{A}$ and such that the $\mathscr{Z}$-linear functions $\phi_{1}(A)=\phi(A E)$ and $\phi_{2}(A)=-\phi(A(1-E))$ of $\mathscr{A}$ are normal.

Proof. Let $x$ be a nonzero vector in $H$. The linear functional $\rho(A)=(\phi(A) x, x)$ on $\mathscr{A}$ is a nonzero ultraweakly continuous hermitian linear functional. There is a projection $F$ in $\mathscr{A}$ such that $\rho(F A)=\rho(A F)$ for every $A$ in $\mathscr{A}$ and such that $A \rightarrow \rho(A F)$ 
and $A \rightarrow-\rho(A(1-F))$ are normal functionals on $\mathscr{A}$. For every $B$ in $\mathscr{Z}$ and $A$ in $\mathscr{A}^{+}$ we have that

$$
0 \leqq\left(\phi\left(A F B^{*} B\right) x, x\right)=(\phi(A F) B x, B x)=(\phi(F A) B x, B x),
$$

and

$$
0 \leqq-\left(\phi(A(1-F)) B^{*} B x, x\right)=-(\phi(A(1-F)) B x, B x) .
$$

Let $G^{\prime}$ be nonzero projection in the commutator $\mathscr{Z}^{\prime}$ of $\mathscr{Z}$ that corresponds to the subspace closure $\{B x \mid B \in \mathscr{Z}\}$. Since $0 \leqq(\phi(A F) y, y)=(\phi(F A) y, y)$ and $0 \leqq-(\phi(A(1-F)) y, y)$ for every $A$ in $\mathscr{A}^{+}$and $y$ in closure $\{B x \mid B \in \mathscr{Z}\}$, we have that $\phi(A F) G^{\prime}=\phi(F A) G^{\prime}$ and $-\phi(A(1-F)) G^{\prime}$ are positive central elements in $G^{\prime} \mathscr{Z}^{\prime} G^{\prime}$ for every $A$ in $\mathscr{A}^{+}$. Let $G$ be the projection in $\mathscr{Z}$ corresponding to closure $\left\{B^{\prime} x \mid B^{\prime} \in \mathscr{Z}^{\prime}\right\}$. The function $A \rightarrow A G^{\prime}$ of $\mathscr{Z} G$ onto $\mathscr{Z} G^{\prime}$ is an isomorphism. So $\phi(A F) G=\phi(F A) G$ and $-\phi(A(1-F)) G$ are positive operators for each $A$ in $\mathscr{A}^{+}$.

Now let $\left\{G_{n}\right\}$ be a maximal net of nonzero orthogonal central projections such that for each $n$ there is a projection $E_{n}$ majorized by $G_{n}$ such that $\phi\left(A E_{n}\right)=\phi\left(E_{n} A\right)$ and $-\phi\left(A\left(G_{n}-E_{n}\right)\right)$ are positive for each $A$ in $\mathscr{A}^{+}$. Let $G=\sum G_{n}$ and $E=\sum E_{n}$. Then $\phi(A E)=\phi(E A)$ and $-\phi(A(G-E))$ are positive for each $A$ in $\mathscr{A}^{+}$due to the ultraweak continuity of $\phi$. From the preceding paragraph we see that the maximality of $\left\{G_{n}\right\}$ implies that $G=1$. Q.E.D.

We now identify the elements of trace class with the ultraweakly continuous $\mathscr{Z}$-linear functions of $\mathscr{A}$ into $\mathscr{Z}$.

THEOREM 4.13. Let $\mathscr{A}$ be a Type I von Neumann algebra and let $\mathscr{Z}$ be the center of $\mathscr{A}$. Let $\mathscr{P}$ be the set of positive elements of the trace class $\mathscr{T}$ of $\mathscr{A}$ and let $\operatorname{Tr}$ be the trace function. If $A \in \mathscr{T}$, let $\phi_{A}$ be the function on $\mathscr{A}$ defined by $\phi_{A}(B)=\operatorname{Tr}(B A)$. The function $A \rightarrow \phi_{A}$ is an isometric isomorphism of $\mathscr{T}$ onto the set of all ultraweakly continuous $\mathscr{Z}$-linear functions of $\mathscr{A}$ into $\mathscr{Z}$. The function $A \rightarrow \phi_{A}$ takes $\mathscr{P}$ onto the set of all normal $\mathscr{Z}$-linear functions.

Proof. Let $\phi$ be an ultraweakly continuous $\mathscr{Z}$-linear function of $\mathscr{A}$ into $\mathscr{Z}$. By Theorem 4.12 and the remarks preceding it the function $\phi$ may be written as a linear combination of four normal $\mathscr{Z}$-linear functions $\phi_{j}(1 \leqq j \leqq 4)$. There is a constant $K$ such that

$$
\left\|\phi_{j}(B) \phi_{j}(B) *\right\| \leqq K\left\|\phi_{j}\left(B^{*} B\right)\right\| \leqq K\left\|\phi_{j}(1)\right\|\|B\|^{2}
$$

for every $B$ in $\mathscr{A}$. Therefore, the functions $\phi_{j}$ restricted to $I_{a}$ are uniformly continuous $\mathscr{Z}$-linear functionals. There are unique elements $A_{j}$ in $\mathscr{T}$ such that $\phi_{j}(B)$ $=\operatorname{Tr}\left(B A_{j}\right)$ for each $B$ in $I_{a}$. For every abelian projection $E$ in $\mathscr{A}$ we have that $0 \leqq \phi_{j}(E)=\tau_{E}\left(A_{j}\right)$. This shows that each $A_{j}$ is an element of $\mathscr{P}$.

Finally, the functions $\theta_{j}(B)=\operatorname{Tr}\left(B A_{j}\right)$ are normal on $\mathscr{A}$. However, the ideal $I_{a}$ is ultraweakly dense in $\mathscr{A}$. Since $\theta_{j}$ is equal to $\phi_{j}$ on a dense subset of $\mathscr{A}$, we have that $\theta_{j}$ is equal to $\phi_{j}$ for each $j$. Thus the function $A \rightarrow \phi_{A}$ of $\mathscr{T}$ maps onto the set of ultraweakly continuous $\mathscr{Z}$-linear functions of $\mathscr{A}$ into $\mathscr{Z}$ and maps $\mathscr{P}$ onto the 
set of normal $\mathscr{Z}$-linear functions. This function is certainly a monomorphism. Furthermore, by Theorem 4.9 we have $\|\operatorname{Tr}(|A|)\|=\operatorname{lub}\left\{\left\|\phi_{A}(B)\right\| \mid\|B\| \leqq 1, B \in I_{a}\right\}$. By the Kaplansky density theorem [6] the unit sphere of $I_{a}$ is strongly dense in the unit sphere of $\mathscr{A}$. Let $\varepsilon>0$ be given; then there is an operator $B$ in the unit sphere of $\mathscr{A}$ and unit vectors $x$ and $y$ in the Hilbert space $H$ of $\mathscr{A}$ such that $\left\|\phi_{A}\right\|$ $\leqq\left|\left(\phi_{A}(B) x, y\right)\right|+\varepsilon$. Therefore, there is an element $C$ in the unit sphere of $I_{a}$ such that $\left\|\phi_{A}\right\| \leqq\left|\left(\phi_{A}(C) x, y\right)\right|+2 \varepsilon$. So $\left\|\phi_{A}\right\| \leqq\left|\left(\phi_{A}(C) x, y\right)\right|+2 \varepsilon \leqq\|\operatorname{Tr}(|A|)\|+2 \varepsilon$. Because $\varepsilon>0$ is arbitrary, we have that $\left\|\phi_{A}\right\| \leqq\|\operatorname{Tr}(|A|)\|$. Finally, we obtain that $\left\|\phi_{A}\right\|$ $=\|\operatorname{Tr}(|A|)\|$. Therefore, the function $A \rightarrow \phi_{A}$ is an isometric isomorphism of $\mathscr{T}$ onto the space of all ultraweakly continuous $\mathscr{Z}$-linear functions of $\mathscr{A}$ into $\mathscr{Z}$. Q.E.D.

Let $\mathscr{A}$ be a semifinite von Neumann algebra with center $\mathscr{Z}$. Let $X$ be a locally compact topological space and let $v$ be a positive measure on $X$ so that the set of all essentially bounded measurable functions $L_{C}^{\infty}(X, v)$ on $X$ is isometrically isomorphic to $\mathscr{Z}$. Let $\Phi$ be a normal semifinite faithful \#-map of $\mathscr{A}^{+}$into the set of all positive (finite or infinite valued) measurable functions on $X$. Let $\mathscr{N}=\left\{A \in \mathscr{A} \mid \Phi\left(A^{*} A\right) \in \mathscr{Z}\right\}$ and $\mathscr{M}=\mathscr{N}^{2}$. For $A$ and $B \in \mathscr{N}$, let $(A, B)=\Phi\left(B^{*} A\right)$ and let $\|A\|_{1}=\left\|\Phi\left(A^{*} A\right)^{1 / 2}\right\|$. The function $A \rightarrow\|A\|_{1}$ on $\mathscr{N}$ is a norm. The following theorem gives part of our reason for not studying the subject of the preceding section in a more general context.

THEOREM 4.14. Let $\Phi$ be a normal semifinite faithful \#-map on the von Neumann algebra $\mathscr{A}$ with center $\mathscr{Z}$; then the ideal $\mathscr{N}=\left\{A \in \mathscr{A} \mid \Phi\left(A^{*} A\right) \in \mathscr{Z}\right\}$ is complete under the norm $\|A\|_{1}=\left\|\Phi\left(A^{*} A\right)^{1 / 2}\right\|$ if and only if there is an $\alpha>0$ such that $\|A\|$ $\leqq \alpha\|A\|_{1}$ for all $A \in \mathscr{N}$. In particular $\mathscr{A}$ is of Type $\mathrm{I}$.

Proof. Let $\mathscr{N}$ be complete under the norm $\|A\|_{1}$. There is no loss of generality in assuming either that $\mathscr{A}$ is of Type I or that $\mathscr{A}$ is of Type II. Suppose first that $\mathscr{A}$ is of Type I and there is no $\alpha>0$ such that $\|A\| \leqq \alpha\|A\|_{1}$ for all $A \in \mathscr{N}$. There is a sequence $\left\{A_{n}\right\}$ of elements of $\mathscr{N}$ such that $\left\|A_{n}\right\|=1$ and $\left\|A_{n}\right\|_{1}<2^{-(2 n+1)}$. We may assume that $A_{n} \in \mathscr{A}^{+}$for each $n$. There is for each $n$ a nonzero abelian projection $E_{n}$ such that $A_{n}^{2} \geqq 2^{-1} E_{n}$. Thus

$$
\left\|E_{n}\right\|_{1}=\left\|\Phi\left(E_{n}\right)^{1 / 2}\right\| \leqq 2^{1 / 2}\left\|\Phi\left(A_{n}^{2}\right)^{1 / 2}\right\| \leqq 2^{-2 n} .
$$

Let $P_{n}$ be the central support of $E_{n}$ and let $X_{n}$ be the set in the spectrum $Z$ of $\mathscr{Z}$ to which $P_{n}$ corresponds. We consider two cases: (1) there is a subsequence $\left\{X_{n}\right\}$ of $\left\{X_{n}\right\}$ such that $\bigcap\left\{X_{n} \mid j=1,2, \ldots\right\} \neq \varnothing ;(2)$ for every subsequence $\left\{X_{n}\right\}$ of $\left\{X_{n}\right\}$, $\bigcap\left\{X_{n j} \mid j=1,2, \ldots\right\}=\varnothing$. In case (1) let $\bigcap\left\{X_{n j} \mid j=1,2, \ldots\right\} \neq \varnothing$. Let $Q_{j}=$ $P_{n_{1}} P_{n_{2}} \cdots P_{n j}$ and let $F_{j}=Q, E_{n_{j}}$. Then $\left\{F_{n}\right\}$ is a sequence of nonzero abelian projections such that $\left\|F_{n}\right\|_{1} \leqq 2^{-2 n}$ and $F_{n+1} \prec F_{n}$. We may assume therefore that $F_{n+1}<F_{n}(n=1,2, \ldots)$ by choosing inductively appropriate projections equivalent to each of the original $F_{n}$ and by passing to a subsequence if necessary. Let $G_{n}$ $=F_{n}-F_{n+1}(n=1,2, \ldots)$. Then $\left\{G_{n}\right\}$ is a sequence of nonzero disjoint abelian 
projections such that $\left\|G_{n}\right\|_{1} \leqq 2^{-2 n}$. Now consider case (2). There is a sequence $\left\{\pi_{j}\right\}$ of nonempty finite disjoint subsets of natural numbers with the following properties: (1) if $i<j$ and $k \in \pi_{i}, l \in \pi_{j}$ then $k<l$; (2) $Q_{j}=\times\left\{P_{k} \mid k \in \pi_{j}\right\} \neq 0$ for each $j$; and (3) if $k>j$ then $P_{l}$ is orthogonal to $Q_{j}$ for each $l \in \pi_{k}$. Let $m(j)$ be the largest integer in $\pi_{j}$ and let $G_{j}=Q_{j} E_{m(j)}$. Then $\left\{G_{n}\right\}$ is a sequence of nonzero disjoint abelian projections such that $\left\|G_{n}\right\|_{1} \leqq 2^{-2 n}$.

Now for either case let $B_{n}=\sum\left\{2^{k} G_{k} \mid 1 \leqq k \leqq n\right\}$ for $n=1,2, \ldots$. Since

$$
\Phi\left(\left(B_{m}-B_{n}\right)^{*}\left(B_{m}-B_{n}\right)\right)^{1 / 2}=\left(\sum\left\{2^{2 j} \Phi\left(G_{j}\right) \mid m+1 \leqq j \leqq n\right\}\right)^{1 / 2}
$$

for $m<n$, we have

$$
\left\|B_{m}-B_{n}\right\|_{1} \leqq\left(\sum\left\{2^{2 j}\left\|\Phi\left(G_{j}\right)\right\| \mid m+1 \leqq j \leqq n\right\}\right)^{1 / 2} \leqq 2^{-m} .
$$

Thus, the sequence $\left\{B_{n}\right\}$ is Cauchy in $\mathscr{N}$. By assumption there is a $B$ in $\mathscr{N}$ such that $\lim _{n}\left\|B_{n}-B\right\|_{1}=0$. However, the sequence $\left\{B_{n} \sum\left(G_{k} \mid 1 \leqq k \leqq m\right)\right\}_{n}$ which is eventually constant converges to $\sum\left(G_{k} \mid 1 \leqq k \leqq m\right) B$ in $\mathscr{N}$. So $\sum\left(G_{k} \mid 1 \leqq k \leqq m\right) B$ $=\sum\left(2^{k} G_{k} \mid 1 \leqq k \leqq m\right)$ and hence $\|B\| \geqq 2^{m}$. Because $m$ is arbitrary this is impossible. We have proved that if $\mathscr{A}$ is of Type $\mathrm{I}$, and $\mathscr{N}$ is complete there is an $\alpha>0$ such that $\|A\| \leqq \alpha\|A\|_{1}$ for every $A$ in $\mathscr{N}$.

Let $\mathscr{A}$ be a Type II algebra and let $E$ be a nonzero projection in $\mathscr{N}$. Let $F_{01}=E$ and let $\left\{F_{n j} \mid n=0,1, \ldots ; 1 \leqq j \leqq 16\right\}$ be projections in $\mathscr{A}$ such that $\left\{F_{n j} \mid 1 \leqq j \leqq 16\right\}$ is a set of orthogonal equivalent projections of sum $F_{n-11}$, for each $n=1,2, \ldots$ Then $\Phi\left(F_{n-11}\right)=\sum_{j} \Phi\left(F_{n j}\right)=16 \Phi\left(F_{n 1}\right)$. Let $B_{n}=\sum\left\{2^{j} F_{j 2} \mid 1 \leqq j \leqq n\right\}$. We have that $\left\{B_{n}\right\}$ is a Cauchy sequence in $\mathscr{N}$. Indeed

$$
\begin{aligned}
\left\|B_{m}-B_{n}\right\|_{1} & =\left\|\left[\sum\left\{2^{2 j} \Phi\left(F_{j 2}\right) \mid m+1 \leqq j \leqq n\right\}\right]^{1 / 2}\right\| \\
& =\left\|\sum\left\{2^{2 j} 2^{-4 j} \Phi(E) \mid m+1 \leqq j \leqq n\right\}\right\|^{1 / 2} \\
& \leqq 2^{-m}\|\Phi(E)\|^{1 / 2}
\end{aligned}
$$

whenever $m<n$. Now there is a $B \in \mathscr{N}$ such that $\lim _{n} B_{n}=B$ in $\mathscr{N}$. However, the sequence $\left\{B_{n} \sum\left\{F_{j 2} \mid 1 \leqq j \leqq m\right\}\right\}_{n}$ which is eventually constant converges to $\sum\left\{F_{j 2} \mid 1 \leqq j \leqq m\right\} B$. So $\sum\left\{2^{j} F_{j 2} \mid 1 \leqq j \leqq m\right\}=\sum\left\{F_{j 2} \mid 1 \leqq j \leqq m\right\} B$. Thus $\|B\| \geqq 2^{m}$. This is impossible. Hence $\mathscr{A}$ cannot be of Type II.

Conversely, suppose there is an $\alpha>0$ such that $\|A\| \leqq \alpha\|A\|_{1}$ for every $A \in \mathscr{N}$. Let $\left\{A_{n}\right\}$ be a Cauchy sequence in $\mathscr{N}$. We show that $\left\{A_{n}\right\}$ converges to an element $A$ in $\mathscr{A}$ using the method of Theorem 4.6. Because $\left\{A_{n}\right\}$ is Cauchy, the sequence $\left\{A_{n}\right\}$ converges uniformly to $A \in \mathscr{A}$. Let $S$ be a set of orthogonal abelian projections in $\mathscr{N}$ with least upper bound 1. Let $\pi$ be a finite subset of $S$. Then the relation

$$
\begin{aligned}
& {\left[\sum\left\{\tau_{F}\left(\left(A-A_{n}\right)^{*}\left(A-A_{n}\right)\right) \Phi(F) \mid F \in \pi\right\}\right]^{1 / 2} } \\
& \leqq {\left[\sum\left\{\tau_{F}\left(\left(A-A_{m}\right)^{*}\left(A-A_{m}\right)\right) \Phi(F) \mid F \in \pi\right\}\right]^{1 / 2} } \\
&+\left[\sum\left\{\tau_{F}\left(\left(A_{m}-A_{n}\right)^{*}\left(A_{m}-A_{n}\right)\right) \Phi(F) \mid F \in \pi\right\}\right]^{1 / 2},
\end{aligned}
$$


which is obtained in a manner entirely similar to that in which the corresponding formula of Theorem 4.5 is obtained, gives

$$
\left\|\left(A-A_{n}\right) F_{\pi}\right\|_{1} \leqq\left\|\left(A-A_{m}\right) F_{\pi}\right\|_{1}+\left\|\left(A_{m}-A_{n}\right) F_{\pi}\right\|_{1}
$$

where $F_{\pi}=\sum\{F \mid F \in \pi\}$. So there is a constant $M$ such that $\left\|\left(A-A_{n}\right) F_{\pi}\right\|_{1} \leqq M$ for every finite subset $\pi$ of $S$. Therefore, $A-A_{n} \in \mathscr{N}$ and consequently $A \in \mathscr{N}$. Furthermore, the relation (1) shows that $\lim _{n}\left\|A-A_{n}\right\|_{1}=0$. Q.E.D.

We now are able to show that the theory obtained by using a normal semifinite faithful \#-map with values in the center is different from the theory obtained by using a trace with values in the scalar field. Let $\phi$ be a normal semifinite faithful trace (with scalar values) on a Type I von Neumann algebra $\mathscr{A}$. Let $\mathscr{N}=$ $\left\{A \in \mathscr{A} \mid \phi\left(A^{*} A\right)<+\infty\right\}$ and let $\mathscr{M}=\mathscr{N}^{2}$. Define the norm on $\mathscr{N}$ by $\|A\|_{1}=\phi\left(A^{*} A\right)^{1 / 2}$. If $\mathscr{N}$ is complete with this norm, a simple reworking of the proof of Theorem 4.14 shows that there is an $\alpha>0$ such that $\|A\| \leqq \alpha\|A\|_{1}$ for all $A \in \mathscr{N}$. By a theorem of Ogasawara and Yoshinago [11, Theorem 5] $\mathscr{A}$ contains minimal abelian projections. (In fact $\mathscr{A}$ is the product of factors.) This is not the situation if $\phi$ is a normal semifinite faithful \#-map with values in the center of $\mathscr{A}$.

THeOREM 4.15. Let $\mathscr{A}$ be a Type I von Neumann algebra with center $\mathscr{Z}$ and let $\Phi$ be a faithful normal semifinite \#-map on $\mathscr{A}$. Let $\mathscr{N}=\left\{A \in \mathscr{A} \mid \Phi\left(A^{*} A\right) \in \mathscr{Z}\right\}$ and let $\mathscr{M}=\mathscr{N}^{2}$. If $\mathscr{N}$ is complete with the norm $\|A\|_{1}=\left\|\Phi\left(A^{*} A\right)^{1 / 2}\right\|$, the ideal $\mathscr{N}$ is contained in $\mathscr{S}$ and the ideal $\mathscr{M}$ is contained in $\mathscr{T}$. There is a $B_{0} \in \mathscr{Z}^{+}$such that $A \rightarrow A B_{0}^{1 / 2}$ is an isometric isomorphism of $\mathscr{S}$ onto $\mathscr{N}$ and $A \rightarrow A B_{0}$ is an isometric isomorphism of $\mathscr{T}$ onto $\mathscr{M}$ with the norm $\|A\|_{2}=\|\Phi(|A|)\|$.

Proof. Let $\alpha>0$ be a number such that $\|A\| \leqq \alpha\|A\|_{1}$ for every $A \in \mathscr{N}$. Let $F$ be a projection in $\mathscr{N}$ and let $P$ be the central support of $F$. We have that $F \in I_{a}$ and $\Phi(F) \geqq \alpha^{\prime} P$, where $\alpha^{\prime}=\alpha^{-2}$. So there is a $B$ in $(\mathscr{Z} P)^{+}$such that $\|B\| \leqq \alpha^{\prime}$ and $B \Phi(F)=P$. Let $\left\{F_{i}\right\}$ be a set of mutually orthogonal abelian projections in $\mathscr{N}$ whose least upper bound $F$ is a maximal abelian projection. Let $P_{i}$ be the central support of $F_{i}$ and let $B_{i} \in\left(\mathscr{Z} P_{i}\right)^{+}$have the property $B_{i} \Phi\left(F_{i}\right)=P_{i}$ and $\left\|B_{i}\right\| \leqq \alpha^{\prime}$. There is a $B_{0} \in \mathscr{Z}^{+}$such that $B_{0} P_{i}=P_{i}$ for each $i$. Because $B_{0} \Phi(F)=\operatorname{Tr}(F)$, $B_{0} \Phi(A)=\operatorname{Tr}(A)$ for every $A \in \mathscr{A}^{+}$[2, III, $\S 4$, Theorem 2]. If $E$ is any abelian projection in $\mathscr{N}$, then $B_{0} \Phi(E)=P$ where $P$ is the central support of $E$. Thus, $B_{0}^{1 / 2} \Phi(E)^{1 / 2}=P$. Thus $A \in \mathscr{S}$ implies $A B_{0}^{1 / 2} \in \mathscr{N}$ and $\Phi\left(\left(A B_{0}^{1 / 2}\right)^{*}\left(A B_{0}^{1 / 2}\right)\right)^{1 / 2}=$ $\operatorname{Tr}\left(A^{*} A\right)^{1 / 2}$. Also if $A \in \mathscr{T}$ then $A B_{0} \in \mathscr{M}$ and $\Phi\left(\left|A B_{0}\right|\right)=\operatorname{Tr}(|A|)$. So $A \rightarrow A B_{0}^{1 / 2}$ and $A \rightarrow A B_{0}$ are isometric isomorphic functions of $\mathscr{S}$ into $\mathscr{N}$ and $\mathscr{T}$ into $\mathscr{M}$ respectively.

Let $A \in \mathscr{N}^{+}$. Since every projection of $\mathscr{N}$ is an element of $I_{a}$, the element $A$ is a member of $I_{a}$. If $\sum A_{j} E_{j}$ is a spectral resolution for $A$, then $A^{*} A=A^{2}=\sum A_{j}^{2} E_{j}$ and $\Phi\left(A^{*} A\right)=\sum A_{j}^{2} \Phi\left(E_{j}\right)$. Now $\sum A_{j} \Phi\left(E_{j}\right)^{1 / 2} E_{j}$ is an element of $\mathscr{S}$. Indeed, if $B_{n}=\sum\left\{A_{j} \Phi(E)^{1 / 2} E_{j} \mid 1 \leqq j \leqq n\right\}$, the sequence $\left\{B_{n}\right\}$ converges uniformly to an element $B$ in $I_{a}^{+}$which has a spectral resolution $\sum A_{j} \Phi\left(E_{j}\right) E_{j}$. The relation $E_{1}$ 
$>E_{2} \cdots$ implies $\Phi\left(E_{1}\right)^{1 / 2} \geqq \Phi\left(E_{2}\right)^{1 / 2} \geqq \cdots$ and the relation $\alpha\|G\|_{1} \geqq\|G\|$ for every projection $G \in \mathscr{N}$ implies that the product $A, \Phi\left(E_{j}\right)^{1 / 2} E_{j}$ has property (iv) of Theorem 2.2. Also $\lim A_{j} \Phi\left(E_{j}\right)^{1 / 2}=0$. We have that $B B_{0}^{1 / 2}=\sum A_{j} B_{0}^{1 / 2} \Phi\left(E_{j}\right)^{1 / 2} E_{j}$ $=\sum A_{j} E_{j}$. Therefore, the function $A \rightarrow A B_{0}^{1 / 2}$ maps $\mathscr{S}$ onto $\mathscr{N}$.

If $A \in \mathscr{M}^{+}$, then $A$ is an element of $I_{a}$. Let $\sum A_{j} E_{j}$ be a spectral resolution of $A$; we have $\Phi(|A|)=\Phi(A)=\sum A_{j} \Phi(E)$. In the same manner as the preceding paragraph $\sum\left\{A_{j} \Phi\left(E_{j}\right) E_{j} \mid 1 \leqq j \leqq n\right\}$ converges uniformly to an element $B$ in $I_{a}^{+}$whose spectral resolution is $\sum A_{j} \Phi\left(E_{j}\right) E_{j}$. Then $B \in \mathscr{T}$ and $B_{0} B=\sum A_{j} E_{j}$. This shows that the function $A \rightarrow A B_{0}$ of $\mathscr{T}$ into $\mathscr{M}$ is onto $\mathscr{M}$. Q.E.D.

Corollary. If $\mathscr{M}=\mathscr{T}$, then $B_{0}$ is invertible.

\section{BIBLIOGRAPHY}

1. D. Deckard and C. Pearcy, On continuous matrix-valued functions on a Stonian space, Pacific J. Math. 14 (1963), 857-869.

2. J. Dixmier, Les algèbres d'opérateurs dans l'espace hilbertien, Gauthier-Villars, Paris, 1957.

3. - Les $C^{*}$-algèbres et leur représentations, Gauthier-Villars, Paris, 1964.

4. J. Glimm, A Stone-Weierstrass theorem for $C^{*}$-algebras, Ann. of Math. 72 (1960), 216-244.

5. H. Halpern, The maximal GCR ideal in a type I $A W^{*}$-algebra, Proc. Amer. Math. Soc. 17 (1966), 906-914.

6. I. Kaplansky, $A$ theorem on rings of operators, Pacific J. Math. 1 (1951), 227-232.

7. - The structure of certain operator algebras, Trans. Amer. Math. Soc. 70 (1951), 219-225.

8. - Algebras of type I, Ann. of Math. (2) 56 (1952), 460-472.

9. - Modules over operator algebras, Amer. J. Math. 75 (1953), 839-858.

10. J. von Neumann, On some algebraic properties of rings of operators, Ann. of Math. 44 (1943), 709-715.

11. T. Ogasawara and $\mathrm{K}$. Yoshinago, A noncommutative theory of integration for operators, J. Sci. Hiroshima Univ. 18 (1955), 311-347.

12. R. Schatten, Theory of cross spaces, Princeton Univ. Press, Princeton, N. J., 1950.

13. I. E. Segal, Decomposition of operator algebras. I, II, Mem. Amer. Math. Soc. No.9 (1951), 1-67, 1-66.

14. - A noncommutative extension of abstract integration, Ann. of Math. (2) 57 (1953), 401-457.

15. J. Glimm, Type I C*-algebras, Ann. of Math. 73 (1961), 572-612.

ILLINOIS INSTITUTE OF TECHNOLOGY, Chicago, Illinois 